Pacific

Journal of

Mathematics

\title{
EIGENVALUES AND ENTROPIES UNDER THE HARMONIC-RICCI FLOW
}

YI LI

Volume $267 \quad$ No. 1

January 2014 


\title{
EIGENVALUES AND ENTROPIES UNDER THE HARMONIC-RICCI FLOW
}

\author{
YI LI
}

\begin{abstract}
In this paper, the author discusses the eigenvalues and entropies under the harmonic-Ricci flow, which is the Ricci flow coupled with the harmonic map flow. We give an alternative proof of results for compact steady and expanding harmonic-Ricci breathers. In the second part, we derive some monotonicity formulas for eigenvalues of the Laplacian under the harmonic-Ricci flow. Finally, we obtain the first variation of the shrinker and expanding entropies of the harmonic-Ricci flow.
\end{abstract}

1. Introduction 141

2. Notation and commuting identities 148

3. Harmonic-Ricci flow and the evolution equations 150

4. Entropies for harmonic-Ricci flow 150

5. Compact steady harmonic-Ricci breathers 153

6. Compact expanding harmonic-Ricci breathers 154

7. Eigenvalues of the Laplacian under the harmonic-Ricci flow 159

8. Eigenvalues of the Laplacian-type under the harmonic-Ricci flow 166

9. Another formula for $d \lambda(f(t)) / d t \quad 171$

10. The first variation of expander and shrinker entropies $\quad 176$

$\begin{array}{ll}\text { Acknowledgements } & 183\end{array}$

$\begin{array}{ll}\text { References } & 183\end{array}$

\section{Introduction}

Since the successful application of the Ricci flow to topological and geometric problems, several analogous flows have been studied, including the harmonic-Ricci flow [List 2006; Müller 2012], connection Ricci flow [Streets 2008], Ricci-YangMills flow [Streets 2007; 2010; Young 2008], and renormalization group flows [He et al. 2008; Li 2012; Oliynyk et al. 2006; Streets 2009]. In this article, we study the

MSC2010: 53C44, 35K55.

Keywords: Eigenvalue, entropies, harmonic-Ricci flow, harmonic-Ricci breathers. 
eigenvalue problems of the harmonic-Ricci flow, which is the following coupled system:

$$
\begin{aligned}
& \frac{\partial}{\partial t} g(t)=-2 \operatorname{Ric}_{g(t)}+4 d u(t) \otimes d u(t), \\
& \frac{\partial}{\partial t} u(t)=\Delta_{g(t)} u(t) .
\end{aligned}
$$

For convenience, we introduce a new symmetric 2-tensor $\mathscr{S}_{g(t), u(t)}$ whose components $S_{i j}$ are defined by

$$
S_{i j}:=R_{i j}-2 \partial_{i} u \partial_{j} u .
$$

Its trace is $S_{g(t), u(t)}:=g^{i j} S_{i j}=R_{g(t)}-2\left|\nabla_{g(t)} u(t)\right|_{g(t)}^{2}$.

Suppose that $M$ is a compact Riemannian manifold. For any Riemannian metric $g$ and any smooth functions $u, f$, we have a number of functionals:

$$
\begin{aligned}
\mathscr{F}(g, u, f) & =\int_{M}\left(R_{g}+\left|\nabla_{g} f\right|_{g}^{2}-2\left|\nabla_{g} u\right|_{g}^{2}\right) e^{-f} d V_{g}, \\
\mathscr{E}(g, u, f) & =\int_{M}\left(R_{g}-2\left|\nabla_{g} u\right|_{g}^{2}\right) e^{-f} d V_{g}, \\
\mathscr{F}_{k}(g, u, f) & =\int_{M}\left(k R_{g}+\left|\nabla_{g} f\right|_{g}^{2}-2 k\left|\nabla_{g} u\right|_{g}^{2}\right) e^{-f} d V_{g} .
\end{aligned}
$$

List [2006] and Müller [2012] showed that, as in the case of Perelman's $\mathscr{F}$-functional, under the evolution equation

$$
\begin{aligned}
& \frac{\partial}{\partial t} g(t)=-2 \operatorname{Ric}_{g(t)}+4 d u(t) \otimes d u(t), \\
& \frac{\partial}{\partial t} u(t)=\Delta_{g(t)} u(t), \\
& \frac{\partial}{\partial t} f(t)=-\Delta_{g(t)} f(t)-R_{g(t)}+\left|\nabla_{g(t)} f(t)\right|_{g(t)}^{2}+2\left|\nabla_{g(t)} u(t)\right|_{g(t)}^{2},
\end{aligned}
$$

the evolution equation for the $\mathscr{F}$-functional is

$$
\begin{aligned}
\frac{d}{d t} \mathscr{F}(g(t), u(t), f(t)) & =2 \int_{M}\left|\mathscr{Y}_{g(t), u(t)}+\nabla_{g(t)}^{2} f(t)\right|_{g(t)}^{2} e^{-f(t)} d V_{g(t)} \\
+ & 4 \int_{M}\left|\Delta_{g(t)} u(t)-\langle d u(t), d f(t)\rangle_{g(t)}\right|_{g(t)}^{2} e^{-f(t)} d V_{g(t)},
\end{aligned}
$$

which is nonnegative. Based on (1-4), we derive the following.

Theorem 1.1. Under the evolution equation (1-3), one has

$$
\begin{aligned}
& \frac{d}{d t} \mathscr{E}(g(t), u(t), f(t)) \\
& \quad=2 \int_{M}\left|\mathscr{S}_{g(t), u(t)}\right|_{g(t)}^{2} e^{-f(t)} d V_{g(t)}+4 \int_{M}\left|\Delta_{g(t)} u(t)\right|_{g(t)}^{2} e^{-f(t)} d V_{g(t)},
\end{aligned}
$$


and

$$
\begin{aligned}
& \text { (1-6) } \frac{d}{d t} \mathscr{F}_{k}(g(t), u(t), f(t)) \\
& =2(k-1) \int_{M}\left|\mathscr{Y}_{g(t), u(t)}\right|_{g(t)}^{2} e^{-f(t)} d V_{g(t)}+2 \int_{M} \mid \mathscr{Y}_{g(t), u(t)} \\
& +\left.\nabla_{g(t)}^{2} f(t)\right|_{g(t)} ^{2} e^{-f(t)} d V_{g(t)}+4(k-1) \int_{M}\left|\Delta_{g(t)} u(t)\right|_{g(t)}^{2} e^{-f(t)} d V_{g(t)} \\
& +4 \int_{M}\left|\Delta_{g(t)} u(t)-\langle d u(t), d f(t)\rangle_{g(t)}\right|_{g(t)}^{2} e^{-f(t)} d V_{g(t)} .
\end{aligned}
$$

As a corollary we give a new proof of the following result.

Corollary 1.2. There is no compact steady harmonic-Ricci breather unless the manifold $(M, g(t))$ is Ricci-flat and $u(t)$ is a constant.

To deal with the expanding harmonic-Ricci breather, we need the functionals

$$
\begin{gathered}
\mathscr{L}_{+}(g, u, \tau, f)=\tau^{2} \int_{M}\left(R_{g}+\frac{n}{2 \tau}+\Delta_{g} f-2\left|\nabla_{g} u\right|_{g}^{2}\right) e^{-f} d V_{g}, \\
\mathscr{L}_{+, k}(g, u, \tau, f)=\tau^{2} \int_{M}\left(k\left(R_{g}+\frac{n}{2 \tau}\right)+\Delta_{g} f-2 k\left|\nabla_{g} u\right|_{g}^{2}\right) e^{-f} d V_{g} .
\end{gathered}
$$

Under the evolution equation

$$
\begin{aligned}
& \frac{\partial}{\partial t} g(t)=-2 \operatorname{Ric}_{g(t)}+4 d u(t) \otimes d u(t) \\
& \frac{\partial}{\partial t} u(t)=\Delta_{g(t)} u(t) \\
& \frac{\partial}{\partial t} f(t)=-\Delta_{g(t)} f(t)+\left|\nabla_{g(t)} f(t)\right|_{g(t)}^{2}-R_{g(t)}+2\left|\nabla_{g(t)} u(t)\right|_{g(t)}^{2}, \\
& \frac{d}{d t} \tau(t)=1
\end{aligned}
$$

we have:

Theorem 1.3. Under the evolution equation, one has

$$
\begin{aligned}
& \frac{d}{d t} \mathscr{L}_{+}(g(t), u(t), \tau(t), f(t)) \\
& =2 \tau(t)^{2} \int_{M}\left|\mathscr{Y}_{g(t), u(t)}+\nabla_{g(t)}^{2} f(t)+\frac{1}{2 \tau(t)} g(t)\right|_{g(t)}^{2} e^{-f(t)} d V_{g(t)} \\
& \quad+4 \tau(t)^{2} \int_{M}\left|\Delta_{g(t)} u(t)-\langle d u(t), d f(t)\rangle_{g(t)}\right|_{g(t)}^{2} e^{-f(t)} d V_{g(t)}
\end{aligned}
$$


and

$$
\begin{aligned}
& \frac{d}{d t} \mathscr{L}_{+, k}(g(t), u(t), \tau(t), f(t)) \\
& =2 \tau(t)^{2} \int_{M}\left|\mathscr{T}_{g(t), u(t)}+\nabla_{g(t)}^{2} f(t)+\frac{1}{2 \tau(t)} g(t)\right|_{g(t)}^{2} e^{-f(t)} d V_{g(t)} \\
& \quad+2(k-1) \tau(t)^{2} \int_{M}\left|\mathscr{Y}_{g(t), u(t)}+\frac{1}{2 \tau(t)} g(t)\right|_{g(t)}^{2} e^{-f(t)} d V_{g(t)} \\
& +4 \tau(t)^{2} \int_{M}\left|\Delta_{g(t)} u(t)-\langle d u(t), d f(t)\rangle_{g(t)}\right|_{g(t)}^{2} e^{-f(t)} d V_{g(t)} \\
& \quad+4(k-1) \tau(t)^{2} \int_{M}\left|\Delta_{g(t)} u(t)\right|_{g(t)}^{2} e^{-f(t)} d V_{g(t)}
\end{aligned}
$$

As a corollary, we obtain a new proof of the following.

Corollary 1.4. There is no expanding harmonic-Ricci breather on compact Riemannian manifolds unless the manifold $M$ is an Einstein manifold and $u(t) a$ constant.

The second part of this paper focuses on the eigenvalue of the Laplacian operator under the harmonic-Ricci flow.

Theorem 1.5. If $(g(t), u(t))$ is a solution of the harmonic-Ricci flow on a compact Riemannian manifold $M$ and $\lambda(t)$ denotes the eigenvalue of the Laplacian $\Delta_{g(t)}$ with eigenfunction $f(t)$,

$$
\begin{array}{rl}
\frac{d}{d t} \lambda(t) & \cdot \int_{M} f(t)^{2} d V_{g(t)} \\
=\lambda(t) \int_{M} S_{g(t), u(t)} f(t)^{2} & d V_{g(t)}-\int_{M} S_{g(t), u(t)}\left|\nabla_{g(t)} f\right|_{g(t)}^{2} d V_{g(t)} \\
& +2 \int_{M}\left\langle\varphi_{g(t), u(t)}, d f(t) \otimes d f(t)\right\rangle_{g(t)} d V_{g(t)} .
\end{array}
$$

Equation (1-9) is a general formula to describe the evolution of $\lambda(t)$ under the harmonic-Ricci flow. Under a curvature assumption, we can derive some monotonicity formulas for the eigenvalue $\lambda(t)$. Set

$$
S_{\min }(0):=\min _{x \in M} S_{g(0), u(0)}(x),
$$

the minimum of $S_{g(t), u(t)}$ over $M$ at the time 0 .

Theorem 1.6. Let $(g(t), u(t))_{t \in[0, T]}$ be a solution of the harmonic-Ricci flow on a compact Riemannian manifold $M$ and let $\lambda(t)$ denote the eigenvalue of the Laplacian $\Delta_{g(t)}$. Suppose that $\mathscr{Y}_{g(t), u(t)}-\alpha S_{g(t), u(t)} g(t) \geq 0$ along the harmonic-Ricci flow for some $\alpha \geq \frac{1}{2}$. 
(1) If $S_{\min }(0) \geq 0, \lambda(t)$ is nondecreasing along the harmonic-Ricci flow for any $t \in[0, T]$.

(2) If $S_{\min }(0)>0$, the quantity

$$
\left(1-\frac{2}{n} S_{\min }(0) t\right)^{n \alpha} \lambda(t)
$$

is nondecreasing along the harmonic-Ricci flow for $T \leq n /\left(2 S_{\min }(0)\right)$.

(3) If $S_{\min }(0)<0$, the quantity

$$
\left(1-\frac{2}{n} S_{\min }(0) t\right)^{n \alpha} \lambda(t)
$$

is nondecreasing along the harmonic-Ricci flow for any $t \in[0, T]$.

Corollary 1.7. Let $(g(t), u(t))_{t \in[0, T]}$ be a solution of the harmonic-Ricci flow on a compact Riemannian surface $\Sigma$ and let $\lambda(t)$ denote the eigenvalue of the Laplacian $\Delta_{g(t)}$.

(1) Suppose that $\operatorname{Ric}_{g(t)} \leq \epsilon d u(t) \otimes d u(t)$ where

$$
\epsilon \leq 4 \frac{1-\alpha}{1-2 \alpha}, \quad \alpha>\frac{1}{2}
$$

(i) If $S_{\min }(0) \geq 0, \lambda(t)$ is nondecreasing along the harmonic-Ricci flow for any $t \in[0, T]$.

(ii) If $S_{\min }(0)>0$, the quantity

$$
\left(1-S_{\min }(0) t\right)^{2 \alpha} \lambda(t)
$$

is nondecreasing along the harmonic-Ricci flow for $T \leq 1 / S_{\min }(0)$.

(iii) If $S_{\min }(0)<0$, the quantity

$$
\left(1-S_{\min }(0) t\right)^{2 \alpha} \lambda(t)
$$

is nondecreasing along the harmonic-Ricci flow for any $t \in[0, T]$.

(2) Suppose that

$$
\left|\nabla_{g(t)} u(t)\right|_{g(t)}^{2} g(t) \geq 2 d u(t) \otimes d u(t) .
$$

(i) If $S_{\min }(0) \geq 0, \lambda(t)$ is nondecreasing along the harmonic-Ricci flow for any $t \in[0, T]$.

(ii) If $S_{\min }(0)>0$, the quantity

$$
\left(1-S_{\min }(0) t\right) \lambda(t)
$$

is nondecreasing along the harmonic-Ricci flow for $T \leq 1 / S_{\min }(0)$. 
(iii) If $S_{\min }(0)<0$, the quantity

$$
\left(1-S_{\min }(0) t\right) \lambda(t)
$$

is nondecreasing along the harmonic-Ricci flow for any $t \in[0, T]$.

When we restrict to the Ricci flow, we obtain:

Corollary 1.8. Let $(g(t))_{t \in[0, T]}$ be a solution of the Ricci flow on a compact Riemannian surface $\Sigma$ and let $\lambda(t)$ denote the eigenvalue of the Laplacian $\Delta_{g(t)}$.

(1) If $R_{\min }(0) \geq 0, \lambda(t)$ is nondecreasing along the Ricci flow for any $t \in[0, T]$.

(2) If $R_{\min }(0)>0$, the quantity $\left(1-R_{\min }(0) t\right) \lambda(t)$ is nondecreasing along the Ricci flow for $T \leq 1 / R_{\min }(0)$.

(3) If $R_{\min }(0)<0$, the quantity $\left(1-R_{\min }(0) t\right) \lambda(t)$ is nondecreasing along the Ricci flow for any $t \in[0, T]$.

Remark 1.9. Let $(g(t))_{t \in[0, T]}$ be a solution of the Ricci flow on a compact Riemannian surface $\Sigma$ with nonnegative scalar curvature and let $\lambda(t)$ denote the eigenvalue of the Laplacian $\Delta_{g(t)}$. Then $\lambda(t)$ is nondecreasing along the Ricci flow for any $t \in[0, T]$.

Since

$$
\mu(g, u):=\inf \left\{\mathscr{F}(g, u, f) \mid f \in C^{\infty}(M), \int_{M} e^{-f} d V_{g}=1\right\}
$$

is the smallest eigenvalue of the operator $\Delta_{g, u}:=-4 \Delta_{g}+R_{g}-2\left|\nabla_{g} u\right|_{g}^{2}$, we can consider the evolution equation for this eigenvalue under the harmonic-Ricci flow. To the operator $\Delta_{g, u}$ we associate a functional

$$
\lambda_{g, u}(f):=\int_{M} f \Delta_{g, u} f d V_{g} .
$$

When $f$ is an eigenfunction of the operator $\Delta_{g, u}$ with the eigenvalue $\lambda$ and normalized by $\int_{X} f^{2} d V_{g}=1$, we obtain $\lambda_{g, u}(f)=\lambda$. Hence it suffices to study the evolution equation for $(d / d y) \lambda_{g, u}(f)$ under the harmonic-Ricci flow.

Theorem 1.10. Suppose that $(g(t), u(t))$ is a solution of the harmonic-Ricci flow on a compact Riemannian manifold $M$ and $f(t)$ is an eigenfunction of $\Delta_{g(t), u(t)}$, that is, $\Delta_{g(t), u(t)} f(t)=\lambda(t) f(t)$ (where $\lambda(t)$ is only a function of time $t$ ), with the normalized condition $\int_{M} f(t)^{2} d V_{g(t)}=1$. Then we have

$$
\begin{aligned}
\frac{d}{d t} \lambda(t)=\frac{d}{d t} \lambda_{g, u}(f(t)) & =\int_{M} 2\left\langle\mathscr{S}_{g(t), u(t)}, d f(t) \otimes d f(t)\right\rangle_{g(t)} d V_{g(t)} \\
+ & \int_{M} f(t)^{2}\left(\left|\mathscr{Y}_{g(t), u(t)}\right|_{g(t)}^{2}+2\left|\Delta_{g(t)} u(t)\right|_{g(t)}^{2}\right) d V_{g(t)} .
\end{aligned}
$$


List [2006] proved the nonnegativity of the operator $\mathscr{S}_{g(t), u(t)}$ is preserved by the harmonic-Ricci flow. Hence we get the following.

Corollary 1.11. If $\operatorname{Ric}_{g(0)}-2 d u(0) \otimes d u(0) \geq 0$, the eigenvalues of the operator $\Delta_{g(t), u(t)}$ are nondecreasing under the harmonic-Ricci flow.

Remark 1.12. If we choose $u(t) \equiv 0$, we obtain X. Cao's result [2007].

There is another expression for $d \lambda(t) / d t$.

Theorem 1.13. Suppose that $(g(t), u(t))$ is a solution of the harmonic-Ricci flow on a compact Riemannian manifold $M$ and $f(t)$ is an eigenfunction of $\Delta_{g(t), u(t)}$, that is, $\Delta_{g(t), u(t)} f(t)=\lambda(t) f(t)$ (where $\lambda(t)$ is only a function of time $t$ ), with the normalized condition $\int_{M} f(t)^{2} d V_{g(t)}=1$. Then we have

$$
\begin{aligned}
& \frac{d}{d t} \lambda(t)=\frac{d}{d t} \lambda_{g, u}(f(t))=\frac{1}{2} \int_{M}\left|\mathscr{S}_{g(t), u(t)}+\nabla_{g(t)}^{2} \varphi(t)\right|_{g(t)}^{2} e^{-\varphi(t)} d V_{g(t)} \\
& +\frac{1}{4} \int_{M}\left|\mathscr{S}_{g(t), u(t)}\right|_{g(t)}^{2} e^{-\varphi(t)} d V_{g(t)}+\int_{M}\left|\langle d u(t), d \varphi(t)\rangle_{g(t)}\right|^{2} e^{-\varphi(t)} d V_{g(t)} \\
& +2 \int_{M}\left|\nabla_{g(t)}^{2} u(t)\right|_{g(t)}^{2} e^{-\varphi(t)} d V_{g(t)}-\int_{M} \Delta_{g(t)}\left(\left|\nabla_{g(t)} u(t)\right|_{g(t)}^{2}\right) e^{-\varphi(t)} d V_{g(t)} \\
& +\frac{1}{4} \int_{M}\left|\mathscr{Y}_{g(t), u(t)}+4 d u(t) \otimes d u(t)\right|_{g(t)}^{2} e^{-\varphi(t)} d V_{g(t)},
\end{aligned}
$$

where $f(t)^{2}=e^{-\varphi(t)}$.

Remark 1.14. When $u \equiv 0$, (1-14) reduces to J. Li's formula [2007].

Suppose that $M$ is a compact manifold of dimension $n$. For any Riemannian metric $g$, any smooth functions $u, f$, and any positive number $\tau$, we define

$$
\mathcal{W}_{ \pm}(g, u, f, \tau):=\int_{M}\left[\tau\left(S_{g}+\left|\nabla_{g} f\right|_{g}^{2}\right) \mp f \pm n\right] \frac{e^{-f}}{(4 \pi \tau)^{n / 2}} d V_{g} .
$$

Set

$$
\begin{gathered}
\mu_{ \pm}(g, u, \tau):=\inf \left\{W_{ \pm}(g, u, f, \tau) \mid f \in C^{\infty}(M), \int_{M} \frac{e^{-f}}{(4 \pi \tau)^{n / 2}} d V_{g}=1\right\} \\
\nu_{-}(g, u):=\inf \left\{\mu_{-}(g, u, \tau) \mid \tau>0\right\}, \quad \nu_{+}(g, u):=\sup \left\{\mu_{+}(g, u, \tau) \mid \tau>0\right\} .
\end{gathered}
$$

The first variation of $\nu_{ \pm}(g(s), u(s))$ is the following.

Theorem 1.15. Suppose that $(M, g)$ is a compact Riemannian manifold and $u$ a smooth function on $M$. Let $h$ be any symmetric covariant 2-tensor on $M$ and set $g(s):=g+s h$. Let $v$ be any smooth function on $M$ and $u(s):=u+s v$. If 
$v_{ \pm}(g(s), u(s))=\mathscr{W}_{ \pm}\left(g(s), u(s), f_{ \pm}(s), \tau_{ \pm}(s)\right)$ for some smooth functions $f_{ \pm}(s)$ with $\int_{M} e^{-f_{ \pm}(s)} d V_{g} /\left(4 \pi \tau_{ \pm}(s)\right)^{n / 2}=1$ and constants $\tau_{ \pm}(s)>0$,

(1-16) $\left.\frac{d}{d s}\right|_{s=0} \nu_{ \pm}(g(s), u(s))=4 \tau_{ \pm} \int_{M} v\left(\Delta_{g} u-\left\langle d u, d f_{ \pm}\right\rangle_{g}\right) \frac{e^{-f_{ \pm}}}{\left(4 \pi \tau_{ \pm}\right)^{n / 2}} d V_{g}$

$$
-\tau_{ \pm} \int_{M}\left(\left\langle h, \mathscr{Y}_{g, u}\right\rangle_{g}+\left\langle h, \nabla_{g}^{2} f\right\rangle_{g} \pm \frac{\operatorname{tr}_{g} h}{2 \tau_{ \pm}}\right) \frac{e^{-f_{ \pm}} d V_{g}}{\left(4 \pi \tau_{ \pm}\right)^{n / 2}},
$$

where $f_{ \pm}:=f_{ \pm}(0)$ and $\tau_{ \pm}:=\tau_{ \pm}(0)$. In particular, the critical points of $v_{ \pm}(\cdot, \cdot)$ satisfy

$$
\mathscr{Y}_{g, u}+\nabla_{g}^{2} f \pm \frac{1}{2 \tau_{ \pm}} g=0, \quad \Delta_{g} u=\left\langle d u, d f_{ \pm}\right\rangle_{g} .
$$

Consequently, if $W_{ \pm}(g, u, f, \tau)$ and $\nu_{ \pm}(g, u)$ achieve their extremum, $(M, g)$ is a gradient expanding and shrinker harmonic-Ricci soliton according to the sign.

Corollary 1.16. Suppose that $(M, g)$ is a compact Riemannian manifold and $u$ a smooth function on $M$. Let $h$ be any symmetric covariant 2-tensor on $M$ and set $g(s):=g+s h$. Let $v$ be any smooth function on $M$ and $u(s):=u+s v$. If $\nu_{ \pm}(g(s), u(s))=W_{ \pm}\left(g(s), u(s), f_{ \pm}(s), \tau_{ \pm}(s)\right)$ for some smooth function $f_{ \pm}(s)$ with $\int_{M} e^{-f_{ \pm}(s)} d V /\left(4 \pi \tau_{ \pm}(s)\right)^{n / 2}=1$ and a constant $\tau_{ \pm}(s)>0$, and $(g, u)$ is a critical point of $v_{ \pm}(\cdot, \cdot)$, then

$$
\operatorname{Ric}_{g}=\mp \frac{1}{2 \tau_{ \pm}} g, \quad f_{ \pm} \equiv \text { constant }, \quad u \equiv \text { constant }
$$

Thus, if $\mathcal{W}_{ \pm}(g, u, \cdot, \cdot)$ achieve their minimum and $(g, u)$ is a critical point of $v_{ \pm}(\cdot, \cdot),(M, g)$ is an Einstein manifold and $u$ is a constant function.

Remark 1.17. In the situation of Corollary 1.16 , by normalization, we my choose $f_{ \pm}=n / 2$ and $u=0$.

\section{Notation and commuting identities}

Let $M$ be a compact Riemannian manifold of dimension $n$. For any vector bundle $E$ over $M$, we denote by $\Gamma(M, E)$ the space of smooth sections of $E$. Set

$$
\begin{aligned}
\bigodot^{2}(M) & :=\left\{v=\left(v_{i j}\right) \in \Gamma\left(M, T^{*} M \otimes T^{*} M\right) \mid v_{i j}=v_{j i}\right\}, \\
\bigodot_{+}^{2}(M): & =\left\{g=\left(g_{i j}\right) \in \bigodot^{2}(M) \mid g_{i j}>0\right\} .
\end{aligned}
$$

Thus $\bigodot^{2}(M)$ is the space of all symmetric covariant 2-tensors on $M$, while $\bigodot_{+}^{2}(M)$ is the space of all Riemannian metrics on $M$. The space of all smooth functions on $M$ is denoted by $C^{\infty}(M)$. 
For a given Riemannian metric $g \in \bigodot_{+}^{2}(M)$, the corresponding Levi-Civita connection $\Gamma_{g}=\left(\Gamma_{i j}^{k}\right)$ is given by

$$
\Gamma_{i j}^{k}=\frac{1}{2} g^{k \ell}\left(\partial_{i} g_{j \ell}+\partial_{j} g_{i l}-\partial_{\ell} g_{i j}\right)
$$

where $\partial_{i}:=\partial / \partial x^{i}$ for a local coordinate system $\left\{x^{1}, \ldots, x^{n}\right\}$. The Riemann tensor $\mathrm{Rm}_{g}=\left(R_{i j l}^{k}\right)$ is determined by

$$
R_{i j \ell}^{k}=\partial_{i} \Gamma_{j \ell}^{k}-\partial_{j} \Gamma_{i \ell}^{k}+\Gamma_{i p}^{k} \Gamma_{j \ell}^{p}-\Gamma_{j p}^{k} \Gamma_{i \ell}^{p}
$$

The Ricci curvature $\operatorname{Ric}_{g}=\left(R_{i j}\right)$ is

$$
R_{i j}=g^{k \ell} R_{k i j}^{\ell}
$$

The scalar curvature $R_{g}$ of the metric $g$ now is given by

$$
R_{g}=g^{i j} R_{i j}
$$

For any tensor $A=\left(A_{j_{1} \cdots j_{p}}^{k_{1} \cdots k_{q}}\right)$ the covariant derivative of $A$ is

$$
\nabla_{i} A_{j_{1} \cdots j_{p}}^{k_{1} \cdots k_{q}}=\partial_{i} A_{j_{1} \cdots j_{p}}^{k_{1} \cdots k_{q}}-\sum_{r=1}^{p} \Gamma_{i j_{r}}^{m} A_{j_{1} \cdots m \cdots j_{p}}^{k_{1} \cdots k_{q}}+\sum_{s=1}^{q} \Gamma_{i m}^{k_{s}} A_{j_{1} \cdots j_{p}}^{k_{1} \cdots m \cdots k_{q}}
$$

Next we recall the Ricci identity:

$$
\nabla_{i} \nabla_{j} A_{k_{1} \cdots k_{p}}^{\ell_{1} \cdots \ell_{q}}-\nabla_{j} \nabla_{i} A_{k_{1} \cdots k_{p}}^{\ell_{1} \cdots \ell_{q}}=\sum_{r=1}^{q} R_{i j m}^{l_{r}} A_{k_{1} \cdots k_{p}}^{\ell_{1} \cdots m \cdots \ell_{q}}-\sum_{s=1}^{p} R_{i j k_{s}}^{m} A_{k_{1} \cdots m \cdots k_{p}}^{\ell_{1} \cdots \ell_{q}} .
$$

In particular, for any smooth function $f \in C^{\infty}(M)$, we have

$$
\nabla_{i} \nabla_{j} f=\nabla_{j} \nabla_{i} f
$$

The Bianchi identities are

$$
\begin{aligned}
& 0=R_{i j k \ell}+R_{i k l j}+R_{i \ell j k}, \\
& 0=\nabla_{q} R_{i j k \ell}+\nabla_{i} R_{j q k \ell}+\nabla_{j} R_{q i k \ell},
\end{aligned}
$$

and the contracted Bianchi identities are

$$
\begin{aligned}
& 0=2 \nabla^{j} R_{i j}-\nabla_{i} R_{g}, \\
& 0=\nabla_{i} R_{j k}-\nabla_{j} R_{i k}+\nabla^{\ell} R_{\ell k i j} .
\end{aligned}
$$




\section{Harmonic-Ricci flow and the evolution equations}

Motivated by the static Einstein vacuum equation, List [2006] introduced the harmonic-Ricci flow (originally called the Ricci flow coupled with the harmonic map flow). This flow is similar to the Ricci flow and is given by the coupled system

$$
\begin{aligned}
\frac{\partial}{\partial t} g(t) & =-2 \operatorname{Ric}_{g(t)}+4 d u(t) \otimes d u(t), \\
\frac{\partial}{\partial t} u(t) & =\Delta_{g(t)} u(t)
\end{aligned}
$$

for a family of Riemannian metrics $g(t)$ and a family of smooth functions $u(t)$. Locally, we have

$$
\frac{\partial}{\partial t} g_{i j}=-2 R_{i j}+4 \partial_{i} u \cdot \partial_{j} u, \quad \frac{\partial}{\partial t} u=\Delta_{g(t)} u(t) .
$$

Introduce a new symmetric tensor field $\mathscr{Y}_{g(t), u(t)}=\left(S_{i j}\right) \in \bigodot^{2}(M)$,

$$
S_{i j}:=R_{i j}-2 \partial_{i} u \cdot \partial_{j} u .
$$

Then its trace $S_{g(t), u(t)}$ is equal to

$$
S_{g(t), u(t)}=g^{i j} S_{i j}=R_{g(t)}-2\left|\nabla_{g(t)} u(t)\right|_{g(t)}^{2} .
$$

The evolution equation for $R_{g(t)}$ is

$$
\begin{aligned}
\frac{\partial}{\partial t} R_{g(t)}=\Delta_{g(t)} R_{g(t)}+ & 2\left|\operatorname{Ric}_{g(t)}\right|_{g(t)}^{2}+4\left|\Delta_{g(t)} u(t)\right|_{g(t)}^{2} \\
& -4\left|\nabla_{g(t)}^{2} u(t)\right|_{g(t)}^{2}-8\left\langle\operatorname{Ric}_{g(t)}, d u(t) \otimes d u(t)\right\rangle_{g(t)}
\end{aligned}
$$

Also, we have the evolution equation for $\left|\nabla_{g(t)} u\right|_{g(t)}^{2}$,

$$
\text { (3-7) } \frac{\partial}{\partial t}\left|\nabla_{g(t)} u(t)\right|_{g(t)}^{2}=\Delta_{g(t)}\left|\nabla_{g(t)} u(t)\right|_{g(t)}^{2}-2\left|\nabla_{g(t)}^{2} u(t)\right|_{g(t)}^{2}-4\left|\nabla_{g(t)} u(t)\right|_{g(t)}^{4} \text {, }
$$

and the evolution equation for $S_{g(t), u(t)}$,

$$
\frac{\partial}{\partial t} S_{g(t), u(t)}=\Delta_{g(t)} S_{g(t), u(t)}+2\left|\mathscr{Y}_{g(t), u(t)}\right|_{g(t)}^{2}+4\left|\Delta_{g(t)} u(t)\right|_{g(t)}^{2} .
$$

\section{Entropies for harmonic-Ricci flow}

Motivated by Perelman's entropy, List [2006] introduced a similar functional for the harmonic-Ricci flow:

$$
\bigodot_{+}^{2}(M) \times C^{\infty}(M) \times C^{\infty}(M) \rightarrow \mathbb{R}, \quad(g, u, f) \mapsto \mathscr{F}(g, u, f)
$$


where

$$
\mathscr{F}(g, u, f):=\int_{M}\left(R_{g}+\left|\nabla_{g} f\right|_{g}^{2}-2\left|\nabla_{g} u\right|_{g}^{2}\right) e^{-f} d V_{g} .
$$

He also showed that if $(g(t), u(t), f(t))$ satisfies the system

$$
\begin{aligned}
& \frac{\partial}{\partial t} g(t)=-2 \operatorname{Ric}_{g(t)}+4 d u(t) \otimes d u(t)-2 \nabla_{g(t)}^{2} f(t) \\
& \frac{\partial}{\partial t} u(t)=\Delta_{g(t)} u(t)-\langle d u(t), d f(t)\rangle_{g(t)} \\
& \frac{\partial}{\partial t} f(t)=-\Delta_{g(t)} f(t)-R_{g(t)}+2\left|\nabla_{g(t)} u(t)\right|_{g(t)}^{2}
\end{aligned}
$$

the evolution of the entropy is given by

$$
\begin{aligned}
& \frac{d}{d t} \mathscr{F}(g(t), u(t), f(t)) \\
& \quad=2 \int_{M}\left(\left|\mathscr{S}_{g(t), u(t)}+\nabla_{g(t)}^{2} f(t)\right|_{g(t)}^{2}\right. \\
& \left.\quad+2\left|\Delta_{g(t)} u(t)-\langle d u(t), d f(t)\rangle_{g(t)}\right|_{g(t)}^{2}\right) e^{-f(t)} d V_{g(t)} \\
& \quad \geq 0 .
\end{aligned}
$$

Remark 4.1. The system (4-2) is equivalent to

$$
\begin{aligned}
& \frac{\partial}{\partial t} g(t)=-2 \operatorname{Ric}_{g(t)}+4 d u(t) \otimes d u(t), \\
& \frac{\partial}{\partial t} u(t)=\Delta_{g(t)} u(t), \\
& \frac{\partial}{\partial t} f(t)=-\Delta_{g(t)} f(t)-R_{g(t)}+\left|\nabla_{g(t)} f(t)\right|_{g(t)}^{2}+2\left|\nabla_{g(t)} u(t)\right|_{g(t)}^{2} .
\end{aligned}
$$

The same evolution of the entropy holds for system (4-4).

In particular, the entropy is nondecreasing and the equality holds if and only if $(g(t), u(t), f(t))$ satisfies

$$
\begin{aligned}
\mathscr{S}_{g(t), u(t)}+\nabla_{g(t)}^{2} f(t) & =0, \\
\Delta_{g(t)} u(t)-\langle d u(t), d f(t)\rangle_{g(t)} & =0 .
\end{aligned}
$$

Definition 4.2. The $\mathscr{E}$-functional is defined as

$$
\bigodot_{+}^{2}(M) \times C^{\infty}(M) \times C^{\infty}(M) \rightarrow \mathbb{R}, \quad(g, u, f) \mapsto \mathscr{E}(g, u, f),
$$

where

$$
\mathscr{E}(g, u, f):=\int_{M}\left(R_{g}-2\left|\nabla_{g} u\right|_{g}^{2}\right) e^{-f} d V_{g}
$$


Proposition 4.3. Under the evolution equation (4-4), one has

$$
\begin{aligned}
& \frac{d}{d t} \mathscr{E}(g(t), u(t), f(t)) \\
& \quad=2 \int_{M}\left|\mathscr{Y}_{g(t), u(t)}\right|_{g(t)}^{2} e^{-f(t)} d V_{g(t)}+4 \int_{M}\left|\Delta_{g(t)} u(t)\right|_{g(t)}^{2} e^{-f(t)} d V_{g(t)}
\end{aligned}
$$

Proof. Since $S_{g(t), u(t)}=R_{g(t)}-2\left|\nabla_{g(t)} u(t)\right|_{g(t)}^{2}$ and

$$
\begin{aligned}
\frac{\partial}{\partial t} S_{g(t), u(t)} & =\Delta_{g(t)} S_{g(t), u(t)}+2\left|\varphi_{g(t), u(t)}\right|_{g(t)}^{2}+4\left|\Delta_{g(t)} u(t)\right|_{g(t)}^{2}, \\
\frac{\partial}{\partial t} d V_{g(t)} & =-S_{g(t), u(t)} d V_{g(t)},
\end{aligned}
$$

we have

$$
\begin{aligned}
& \frac{d}{d t} \mathscr{E}(g(t), u(t), f(t)) \\
& =\int_{M}\left(\frac{\partial}{\partial t} S_{g(t), u(t)}\right) e^{-f(t)} d V_{g(t)}+\int_{M} S_{g(t), u(t)} \frac{\partial}{\partial t}\left(e^{-f(t)} d V_{g(t)}\right) \\
& =\int_{M}\left(\Delta_{g(t)} S_{g(t), u(t)}+2\left|\mathscr{S}_{g(t), u(t)}\right|_{g(t)}^{2}+4\left|\Delta_{g(t)} u(t)\right|_{g(t)}^{2}\right) e^{-f(t)} d V_{g(t)} \\
& \quad+\int_{M} S_{g(t), u(t)}\left(-\frac{\partial}{\partial t} f(t)-S_{g(t), u(t)}\right) e^{-f(t)} d V_{g(t)} \\
& =2 \int_{M}\left|\mathscr{S}_{g(t), u(t)}\right|_{g(t)}^{2} e^{-f(t)} d V_{g(t)}+4 \int_{M}\left|\Delta_{g(t)} u(t)\right|_{g(t)}^{2} e^{-f(t)} d V_{g(t)} \\
& \quad-\int_{M} S_{g(t), u(t)}\left(\Delta_{g(t)} f(t)-\left|\nabla_{g(t)} f(t)\right|_{g(t)}^{2}+\frac{\partial}{\partial t} f(t)+S_{g(t), u(t)}\right) e^{-f(t)} d V_{g(t)},
\end{aligned}
$$

which implies (4-7).

Definition 4.4. For any $k \geq 1$ we define

$$
\mathscr{F}_{k}(g, u, f):=\int_{M}\left(k R_{g}+\left|\nabla_{g} f\right|_{g}^{2}-2 k\left|\nabla_{g} u\right|_{g}^{2}\right) e^{-f} d V_{g} .
$$

Using the definition, it is easy to show that

$$
\mathscr{F}_{k}(g, u, f)=(k-1) \mathscr{E}(g, u, f)+\mathscr{F}(g, u, f) .
$$

When $k=1$, this is the $\mathscr{F}$-functional. 
Theorem 4.5. Under the evolution equation (4-4), one has

(4-10) $\frac{d}{d t} \mathscr{F}_{k}(g(t), u(t), f(t))$

$$
\begin{aligned}
& =2(k-1) \int_{M}\left|\mathscr{S}_{g(t), u(t)}\right|_{g(t)}^{2} e^{-f(t)} d V_{g(t)}+2 \int_{M} \mid \mathscr{S}_{g(t), u(t)} \\
& +\left.\nabla_{g(t)}^{2} f(t)\right|_{g(t)} ^{2} e^{-f(t)} d V_{g(t)}+4(k-1) \int_{M}\left|\Delta_{g(t)} u(t)\right|_{g(t)}^{2} e^{-f(t)} d V_{g(t)} \\
& \quad+4 \int_{M}\left|\Delta_{g(t)} u(t)-\langle d u(t), d f(t)\rangle_{g(t)}\right|_{g(t)}^{2} e^{-f(t)} d V_{g(t)} .
\end{aligned}
$$

Furthermore, the monotonicity is strict unless $g(t)$ is Ricci-flat, $u(t)$ is constant, and $f(t)$ is constant.

Proof. It immediately follows from (4-3) and (4-7).

Set

$$
\mu_{k}(g, u):=\inf \left\{\mathscr{F}_{k}(g, u, f) \mid f \in C^{\infty}(M), \int_{M} e^{-f} d V_{g}=1\right\}
$$

Then $\mu_{k}(g, u)$ is the lowest eigenvalue of $-4 \Delta_{g}+k\left(R_{g}-2\left|\nabla_{g} u\right|_{g}^{2}\right)$.

\section{Compact steady harmonic-Ricci breathers}

In this section we give an alternative proof on some results on compact steady harmonic-Ricci breathers that were proved in [List 2006; Müller 2012].

Definition 5.1. A solution $(g(t), u(t))$ of the harmonic-Ricci flow (1-1)-(1-2) is called a harmonic-Ricci breather if there exist $t_{1}<t_{2}$, a diffeomorphism $\psi: M \rightarrow M$, and a constant $\alpha>0$ such that

$$
g\left(t_{2}\right)=\alpha \psi^{*} g\left(t_{1}\right), \quad u\left(t_{2}\right)=\psi^{*} u\left(t_{1}\right) .
$$

The cases $\alpha<1, \alpha=1$, and $\alpha>1$, correspond to shrinking, steady, and expanding harmonic-Ricci breathers.

Theorem 5.2. If $(g(t), u(t))$ is a solution of the harmonic-Ricci flow on a compact Riemannian manifold $M$, the lowest eigenvalue $\mu_{k}(g(t), u(t))$ of the operator $-4 \Delta_{g(t)}+k\left(R_{g(t)}-2\left|\nabla_{g(t)} u(t)\right|_{g(t)}^{2}\right)$ is nondecreasing under the harmonic-Ricci flow. The monotonicity is strict unless $g(t)$ is Ricci-flat and $u(t)$ is constant.

Proof. The proof is similar to that given in [Li 2007]. For any $t_{1}<t_{2}$, suppose that

$$
\mu_{k}\left(g\left(t_{2}\right), u\left(t_{2}\right)\right)=\mathscr{F}_{k}\left(g\left(t_{2}\right), u\left(t_{2}\right), f_{k}\left(t_{2}\right)\right)
$$

for some smooth function $f_{k}(x)$. Being an initial value, $f_{k}(x)=f_{k}\left(x, t_{2}\right)$ for some smooth function $f_{k}(x, t)$ satisfying the evolution equation (4-4). The monotonicity 
formula (4-10) implies $\mu_{k}\left(g\left(t_{2}\right), u\left(t_{2}\right)\right) \geq \mathscr{F}_{k}\left(g\left(t_{1}\right), u\left(t_{1}\right), f_{k}\left(t_{1}\right)\right) \geq \mu_{k}\left(g\left(t_{1}\right), u\left(t_{1}\right)\right)$. This completes the proof.

Corollary 5.3. On a compact Riemannian manifold, the lowest eigenvalues of $-\Delta_{g(t)}+(1 / 2)\left(R_{g(t)}-2\left|\nabla_{g(t)} u(t)\right|_{g(t)}^{2}\right)$ are nondecreasing under the harmonicRicciflow.

Proof. Since $\mu_{2}(g(t), u(t)) / 4$ is the lowest eigenvalue of this operator, the result immediately follows from Theorem 5.2.

Corollary 5.4. There is no compact steady harmonic-Ricci breather unless the manifold $(M, g(t))$ is Ricci-flat and $u$ is a constant.

Proof. If $(g(t), u(t))$ is a steady harmonic-Ricci breather, then, for $t_{1}<t_{2}$ given in the definition, we have

$$
\mu_{k}\left(g\left(t_{1}\right), u\left(t_{1}\right)\right)=\mu_{k}\left(g\left(t_{2}\right), u\left(t_{2}\right)\right) .
$$

Hence, using Theorem 5.2, for any $t \in\left[t_{1}, t_{2}\right]$, we must have

$$
\frac{d}{d t} \mu_{k}(g(t), u(t)) \equiv 0 \text {. }
$$

Thus $(M, g(t))$ is Ricci-flat and $u(t)$ is constant.

\section{Compact expanding harmonic-Ricci breathers}

Inspired by [Li 2007], we define a new functional

$$
\bigodot_{+}^{2}(M) \times C^{\infty}(M) \times C^{\infty}(\mathbb{R}) \times C^{\infty}(M) \rightarrow \mathbb{R}, \quad(g, u, \tau, f) \mapsto W_{+}(g, u, \tau, f),
$$

where $(\tau=\tau(t), t \in \mathbb{R})$.

$$
W_{+}(g, u, \tau, f):=\tau^{2} \int_{M}\left(R_{g}+\frac{n}{2 \tau}+\Delta_{g} f-2\left|\nabla_{g} u\right|_{g}^{2}\right) e^{-f} d V_{g} .
$$

Similarly, we define a family of functionals

$$
\mathscr{W}_{+, k}(g, u, \tau, f):=\tau^{2} \int_{M}\left(k\left(R_{g}+\frac{n}{2 \tau}\right)+\Delta_{g} f-2 k\left|\nabla_{g} u\right|_{g}^{2}\right) e^{-f} d V_{g} .
$$

It's clear that $\mathcal{W}_{+, 1}(g, u, \tau, f)=\mathcal{W}_{+}(g, u, \tau, f)$.

Lemma 6.1. One has

$$
\begin{aligned}
\mathscr{W}_{+}(g, u, \tau, f) & =\tau^{2} \mathscr{F}_{k}(g, u, f)+\frac{n}{2} \tau \int_{M} e^{-f} d V_{g}, \\
W_{+, k}(g, u, \tau, f) & =\tau^{2} \mathscr{F}_{k}(g, u, f)+\frac{k n}{2} \tau \int_{M} e^{-f} d V_{g}, \\
W_{+, k}(g, u, \tau, f) & =\mathscr{W}_{+}(g, u, \tau, f)+(k-1)\left(\tau^{2} \mathscr{E}(g, u, f)+\frac{n}{2} \tau \int_{M} e^{-f} d V_{g}\right) .
\end{aligned}
$$


Proof. Since $\Delta\left(e^{-f}\right)=\left(-\Delta f+|\nabla f|^{2}\right) e^{-f}$, it follows that $\mathscr{W}_{+}(g, u, \tau, f)-\tau^{2} \mathscr{F}(g, u, f)$

$$
\begin{aligned}
& =\frac{n}{2} \tau \int_{M} e^{-f} d V_{g}+\tau^{2} \int_{M}\left(\Delta_{g} f-\left|\nabla_{g} f\right|_{g}^{2}\right) e^{-f} d V_{g} \\
& =\frac{n}{2} \tau \int_{M} e^{-f} d V_{g}+\tau^{2} \int_{M} \Delta_{g}\left(e^{-f}\right) d V_{g}=\frac{n}{2} \tau \int_{M} e^{-f} d V_{g} .
\end{aligned}
$$

We can similarly prove the remaining two relations.

Theorem 6.2. Under the coupled system

$$
\begin{aligned}
& \frac{\partial}{\partial t} g(t)=-2 \operatorname{Ric}_{g(t)}+4 d u(t) \otimes d u(t)-2 \nabla_{g(t)}^{2} f(t), \\
& \frac{\partial}{\partial t} u(t)=\Delta_{g(t)} u(t)-\langle d u(t), d f(t)\rangle_{g(t)}, \\
& \frac{\partial}{\partial t} f(t)=-\Delta_{g(t)} f(t)-R_{g(t)}+2\left|\nabla_{g(t)} u(t)\right|_{g(t)}^{2}, \\
& \frac{d}{d t} \tau(t)=1,
\end{aligned}
$$

the first variation formula for $\mathcal{W}_{+}(g(t), u(t), \tau(t), f(t))$ is

$$
\begin{aligned}
& \frac{d}{d t} W_{+}(g(t), u(t), \tau(t), f(t)) \\
& =2 \tau(t)^{2} \int_{M}\left|\mathscr{Y}_{g(t), u(t)}+\nabla_{g(t)}^{2} f(t)+\frac{1}{2 \tau(t)} g(t)\right|_{g(t)}^{2} e^{-f(t)} d V_{g(t)} \\
& \quad+4 \tau(t)^{2} \int_{M}\left|\Delta_{g(t)} u(t)-\langle d u(t), d f(t)\rangle_{g(t)}\right|_{g(t)}^{2} e^{-f(t)} d V_{g(t)},
\end{aligned}
$$

and the first variation formula for $W_{+, k}(g(t), u(t), \tau(t), f(t))$ is

$$
\begin{aligned}
& \frac{d}{d t} W_{+, k}(g(t), u(t), \tau(t), f(t)) \\
& =2 \tau(t)^{2} \int_{M}\left|\mathscr{S}_{g(t), u(t)}+\nabla_{g(t)}^{2} f(t)+\frac{1}{2 \tau(t)} g(t)\right|_{g(t)}^{2} e^{-f(t)} d V_{g(t)} \\
& \quad+2(k-1) \tau(t)^{2} \int_{M}\left|\mathscr{S}_{g(t), u(t)}+\frac{1}{2 \tau(t)} g(t)\right|_{g(t)}^{2} e^{-f(t)} d V_{g(t)} \\
& \quad+4 \tau(t)^{2} \int_{M}\left|\Delta_{g(t)} u(t)-\langle d u(t), d f(t)\rangle_{g(t)}\right|_{g(t)}^{2} e^{-f(t)} d V_{g(t)} \\
& \quad+4(k-1) \tau(t)^{2} \int_{M}\left|\Delta_{g(t)} u(t)\right|_{g(t)}^{2} e^{-f(t)} d V_{g(t)} .
\end{aligned}
$$

Proof. Under this coupled system, we first observe that

$$
\frac{d}{d t}\left(\int_{M} e^{-f(t)} d V_{g(t)}\right)=0
$$


In fact, from $\frac{\partial}{\partial t} d V_{g(t)}=-S_{g(t), u(t)}-\Delta_{g(t)} f(t) d V_{g(t)}$ we obtain

$$
\begin{aligned}
\frac{d}{d t}\left(\int_{M} e^{-f(t)} d V_{g(t)}\right) & =\int_{M}\left(-\frac{\partial}{\partial t} f(t) \cdot d V_{g(t)}+\frac{\partial}{\partial t} d V_{g(t)}\right) e^{-f(t)} \\
& =\int_{M}\left[\Delta_{g(t)} f(t)+S_{g(t), u(t)}-S_{g(t), u(t)}-\Delta_{g(t)} f(t)\right] e^{-f(t)} d V_{g(t)} \\
& =0 .
\end{aligned}
$$

Lemma 6.1 and the identity (6-5) imply

$$
\begin{aligned}
& \frac{d}{d t} W_{+}(g(t), u(t), \tau(t), f(t)) \\
& =\tau(t)^{2} \frac{d}{d t} \mathscr{F}(g(t), u(t), f(t))+2 \tau(t) \mathscr{F}(g(t), u(t), f(t))+\frac{n}{2} \int_{M} e^{-f(t)} d V_{g(t)} \\
& =2 \tau(t)^{2} \int_{M}\left|\mathscr{S}_{g(t), u(t)}+\nabla_{g(t)}^{2} f(t)\right|_{g(t)}^{2} e^{-f(t)} d V_{g(t)} \\
& \quad+4 \tau(t)^{2} \int_{M}\left|\Delta_{g(t)} u(t)-\langle d u(t), d f(t)\rangle_{g(t)}\right|^{2} e^{-f(t)} d V_{g(t)} \\
& \quad+2 \tau(t) \int_{M}\left(S_{g(t), u(t)}+\left|\nabla_{g(t)} f(t)\right|_{g(t)}^{2}\right) e^{-f(t)} d V_{g(t)}+\frac{n}{2} \int_{M} e^{-f(t)} d V_{g(t)},
\end{aligned}
$$

which is (6-3). Using Lemma 6.1 and the same method, we can prove (6-4).

Remark 6.3. Under the coupled system

$$
\begin{aligned}
& \frac{\partial}{\partial t} g(t)=-2 \operatorname{Ric}_{g(t)}+4 d u(t) \otimes d u(t), \\
& \frac{\partial}{\partial t} u(t)=\Delta_{g(t)} u(t), \\
& \frac{\partial}{\partial t} f(t)=-\Delta_{g(t)} f(t)+\left|\nabla_{g(t)} f(t)\right|_{g(t)}^{2}-R_{g(t)}+2\left|\nabla_{g(t)} u(t)\right|_{g(t)}^{2}, \\
& \frac{d}{d t} \tau(t)=1,
\end{aligned}
$$

the same formulas (6-3) and (6-4) hold for $\mathcal{W}_{+}$and $\mathcal{W}_{+, k}$.

Define

(6-6) $\quad \mu_{+}(g, u, \tau):=\inf \left\{\mathscr{W}_{+}(g, u, \tau, f) \mid f \in C^{\infty}(M), \int_{M} e^{-f} d V_{g}=1\right\}$.

Lemma 6.4. For any $\alpha>0$, one has

$$
\mu_{+}(\alpha g, u, \alpha \tau)=\alpha \mu_{+}(g, u, \tau) .
$$

Proof. Set $\bar{g}:=\alpha g$; then $R_{\bar{g}}=\alpha^{-1} R_{g}, \Delta_{\bar{g}} f=\alpha^{-1} \Delta_{g} f$, and $\left|\nabla_{\bar{g}} u\right|_{\bar{g}}^{2}=\alpha^{-1}\left|\nabla_{g(t)} u\right|_{g}^{2}$. Hence 


$$
\begin{aligned}
\mathscr{W}_{+}(\bar{g}, u, \alpha \tau, f) & =\alpha^{2} \tau^{2} \int_{M}\left(R_{\bar{g}}+\frac{n}{2 \alpha \tau}+\Delta_{\bar{g}} f-2\left|\nabla_{\bar{g}} u\right|_{\bar{g}}^{2}\right) e^{-f} d V_{\bar{g}} \\
& =\alpha \tau^{2} \int_{M}\left(R_{g}+\frac{n}{2 \tau}+\Delta_{g} f-2\left|\nabla_{g(t)} u\right|_{g}^{2}\right) \alpha^{n / 2} e^{-f} d V_{g}
\end{aligned}
$$

Since $f \mapsto f-(n / 2) \ln \alpha$ is one-to-one and onto, by taking the infimum, we derive $\mu_{+}(\alpha g, u, \alpha \tau)=\alpha \mu_{+}(g, u, \tau)$.

Definition 6.5. A solution $(g(t), u(t))$ of the harmonic-Ricci flow is called a harmonic-Ricci soliton if there exists a one-parameter family of diffeomorphisms $\psi_{t}: M \rightarrow M$, satisfying $\psi_{0}=\mathrm{id}_{M}$, and a positive scaling function $\alpha(t)$ such that

$$
g(t)=\alpha(t) \psi_{t}^{*} g(0), \quad u(t)=\psi_{t}^{*} u(0) .
$$

The cases $(\partial / \partial t) \alpha(t)=\dot{\alpha}<0, \dot{\alpha}=0$, and $\dot{\alpha}>0$ correspond to shrinking, steady, and expanding harmonic-Ricci solitons, respectively. If the diffeomorphisms $\psi_{t}$ are generated by a (possibly time-dependent) vector field $X(t)$ that is the gradient of some function $f(t)$ on $M$, the soliton is called a gradient harmonic-Ricci soliton and $f$ is called the potential of the harmonic-Ricci soliton.

Müller [2012] showed that if $(g(t), u(t))$ is a gradient harmonic-Ricci soliton with potential $f$,

$$
\begin{aligned}
& 0=\operatorname{Ric}_{g(t)}-2 d u(t) \otimes d u(t)+\nabla_{g(t)}^{2} f(t)+c g(t), \\
& 0=\Delta_{g(t)} u(t)-\left\langle\nabla_{g(t)} u(t), \nabla_{g(t)} f(t)\right\rangle_{g(t)}
\end{aligned}
$$

for some constant $c$.

Corollary 6.6. There is no expanding breather on compact Riemannian manifolds other than expanding gradient harmonic-Ricci solitons.

Proof. The proof is similar to that given in [Li 2007]. Suppose there is an expanding breather on a compact Riemannian manifold $M$. Then, by definition, we have

$$
g\left(t_{2}\right)=\alpha \Phi^{*} g\left(t_{1}\right), \quad u\left(t_{2}\right)=\Phi^{*} u\left(t_{1}\right)
$$

for some $t_{1}<t_{2}$, where $\Phi$ be a diffeomorphism and the constant $\alpha>1$. Let $f_{+}(x)$ be a smooth function where $\mathcal{W}_{+}\left(g\left(t_{2}\right), u\left(t_{2}\right), \tau\left(t_{2}\right), f\left(t_{2}\right)\right)$ attains its minimum. Then there exists a smooth function $f_{+}(x, t): M \times\left[t_{1}, t_{2}\right] \rightarrow \mathbb{R}$ with initial value $f_{+}\left(x, t_{2}\right)=f_{+}(x)$ that satisfies the coupled system in Remark 6.3. Define a linear function

$$
\tau:\left[t_{1}, t_{2}\right] \rightarrow(0,+\infty), \quad \tau\left(t_{2}\right)=T+t_{2}
$$


where $T$ is a constant. By the monotonicity formula, we have

$$
\begin{aligned}
\mu_{+}\left(g\left(t_{2}\right), u\left(t_{2}\right), \tau\left(t_{2}\right)\right) & =W_{+}\left(g\left(t_{2}\right), u\left(t_{2}\right), \tau\left(t_{2}\right), f_{+}\left(t_{2}\right)\right) \\
& \geq W_{+}\left(g\left(t_{1}\right), u\left(t_{1}\right), \tau\left(t_{1}\right), f_{+}\left(t_{1}\right)\right) \\
& \geq \mu_{+}\left(g\left(t_{1}\right), u\left(t_{1}\right), \tau\left(t_{1}\right)\right) .
\end{aligned}
$$

Lemma 6.4 and the diffeomorphic invariant property of the functionals shows

$$
\mu_{+}\left(g\left(t_{1}\right), u\left(t_{1}\right), \tau\left(t_{1}\right)\right) \leq \alpha \mu_{+}\left(g\left(t_{1}\right), u\left(t_{1}\right), \tau\left(t_{1}\right)\right),
$$

which yields

$$
\mu_{+}\left(g\left(t_{1}\right), u\left(t_{1}\right), \tau\left(t_{1}\right)\right) \geq 0
$$

since $\alpha>1$.

If we impose an additional condition $\tau\left(t_{2}\right)=\alpha \tau\left(t_{1}\right)$ and $\tau\left(t_{1}\right)=T+t_{1}$, we have

$$
\tau(t)=\frac{\alpha\left(t-t_{1}\right)-\left(t-t_{2}\right)}{\alpha-1}, \quad T=\frac{t_{2}-\alpha t_{1}}{\alpha-1} .
$$

Then

$$
\frac{\tau\left(t_{2}\right)^{n / 2}}{V_{g\left(t_{2}\right)}}=\frac{\left[\alpha\left(t_{2}-t_{1}\right) /(\alpha-1)\right]^{n / 2}}{\alpha^{n / 2} V_{g\left(t_{1}\right)}}=\frac{\tau\left(t_{1}\right)^{n / 2}}{V_{g\left(t_{1}\right)}} .
$$

The mean value theorem tells us that there exists a time $\bar{t} \in\left[t_{1}, t_{2}\right]$ with

$$
\begin{aligned}
0 & =\left.\frac{d}{d t}\right|_{t=\bar{t}} \log \frac{\tau(t)^{n / 2}}{V_{g(t)}} \\
& =\frac{V_{g(\bar{t})}}{\tau(\bar{t})^{n / 2}} \cdot \frac{(n / 2) \tau(\bar{t})^{n / 2-1} V_{g(\bar{t})}-\left.\tau(\bar{t})^{n / 2}(d / d t)\right|_{t=\bar{t}} V_{g(t)}}{V_{g(\bar{t})}^{2}} \\
& =\frac{n}{2 \tau(\bar{t})}-\left.\frac{1}{V_{g(\bar{t})}} \frac{\partial}{\partial t}\right|_{t=\bar{t}} V_{g(\bar{t})} .
\end{aligned}
$$

From the evolution equation for the volume element $d V_{g(t)}$, we have

$$
\frac{d}{d t} V_{g(t)}=\int_{M} \frac{\partial}{\partial t} d V_{g(t)}=\int_{M}\left(-S_{g(t), u(t)}-\Delta_{g(t)} f(t)\right) d V_{g(t)}=-\int_{M} S_{g(t), u(t)} d V_{g(t)} .
$$

Putting these together yields

$$
0=\frac{n}{2 \tau(\bar{t})}+\frac{1}{V_{g(\bar{t})}} \int_{M} S_{g(\bar{t}), u(\bar{t})} d V_{g(\bar{t})}=\frac{1}{V_{g(\bar{t})}} \int_{M}\left(S_{g(\bar{t}), u(\bar{t})}+\frac{n}{2 \tau(\bar{t})}\right) d V_{g(\bar{t})} .
$$

If we set $\bar{f}=\log V_{g(\bar{t})}$,

$$
0=\mathscr{W}_{+}(g(\bar{t}), u(\bar{t}), \tau(\bar{t}), \bar{f}) \geq \mu_{+}(g(\bar{t}), u(\bar{t}), \tau(\bar{t})) .
$$


By the monotonicity of $\mu_{+}$we obtain

$$
0 \leq \mu_{+}\left(g\left(t_{1}\right), u\left(t_{1}\right), \tau\left(t_{1}\right)\right) \leq \mu_{+}(g(\bar{t}), u(\bar{t}), \tau(\bar{t})) \leq 0
$$

Hence $\mu_{+}\left(g\left(t_{1}\right), u\left(t_{1}\right), \tau\left(t_{1}\right)\right)=\mu_{+}\left(g\left(t_{2}\right), u\left(t_{2}\right), \tau\left(t_{2}\right)\right)=0$ and $W_{+}=0$ on the interval $\left[t_{1}, t_{2}\right]$. This indicates that the first variation of $\mathcal{W}_{+}$must vanish. So the expanding breather is a gradient soliton, that is,

$$
\mathscr{Y}_{g(t), u(t)}+\nabla_{g(t)}^{2} f(t)+\frac{1}{2 \tau(t)} g(t)=0 .
$$

Moreover, in this case $\Delta_{g(t)} u(t)=\langle d u(t), d f(t)\rangle_{g(t)}$.

Because of (6-7), we define

$$
\mu_{+, k}(g, u, \tau):=\inf \left\{W_{+, k}(g, u, \tau, f) \mid f \in C^{+\infty}(M), \int_{M} e^{-f} d V_{g}=1\right\} .
$$

Due to Lemma 6.4, we still have

$$
\mu_{+, k}(\alpha g, u, \alpha \tau)=\alpha \mu_{+, k}(g, u, \tau) .
$$

Corollary 6.7. If $(g(t), u(t))$ is an expanding harmonic-Ricci breather on compact Riemannian manifolds, $M$ is an Einstein manifold and $u(t)$ is constant.

Proof. Using the same method as in Corollary 6.6 and $\mu_{+, k}$, we can show that the first variation of $\mathcal{W}_{+, k}$ must vanish. Hence, from (6-4), one has

$$
\begin{aligned}
\mathscr{Y}_{g(t), u(t)}+\nabla_{g(t)}^{2} f(t)+\frac{1}{2 \tau(t)} g(t) & =0, \\
\mathscr{Y}_{g(t), u(t)}+\frac{1}{2 \tau(t)} g(t) & =0, \\
\Delta_{g(t)} u(t) & =\langle d u(t), d f(t)\rangle_{g(t)}, \\
\Delta_{g(t)} u(t) & =0 .
\end{aligned}
$$

The above four equations can be reduced to the coupled equation

$$
\mathscr{S}_{g(t), u(t)}+\frac{1}{2 \tau(t)} g(t)=0=\Delta_{g(t)} u(t),
$$

which indicates that $u(t)$ is a constant and $\operatorname{Ric}_{g(t)}=-(1 /(2 \tau(t))) g(t)$.

\section{Eigenvalues of the Laplacian under the harmonic-Ricci flow}

In this section we consider the eigenvalues of the Laplacian $\Delta_{g(t)}$ under the harmonicRicci flow 


$$
\begin{aligned}
\frac{\partial}{\partial t} g(t) & =-2 \operatorname{Ric}_{g(t)}+4 d u(t) \otimes d u(t), \\
\frac{\partial}{\partial t} u(t) & =\Delta_{g(t)} u(t) .
\end{aligned}
$$

Suppose that $\lambda(t)$, which is a function of time $t$ only, is an eigenvalue of the Laplacian $\Delta_{g(t)}$ with an eigenfunction $f(t)=f(x, t)$, that is,

$$
-\Delta_{g(t)} f(t)=\lambda(t) f(t) .
$$

Taking the derivative with respect to $t$, we get

$$
-\left(\frac{\partial}{\partial t} \Delta_{g(t)}\right) f(t)-\Delta_{g(t)}\left(\frac{\partial}{\partial t} f(t)\right)=\left(\frac{d}{d t} \lambda(t)\right) f(t)+\lambda(t) \frac{\partial}{\partial t} f(t) .
$$

Integrating the above equation with $f$ yields

$$
\begin{aligned}
&-\int_{M} f(t)\left(\frac{\partial}{\partial t} \Delta_{g(t)}\right) f(t) d V_{g(t)}-\int_{M} f(t) \Delta_{g(t)}\left(\frac{\partial}{\partial t} f(t)\right) d V_{g(t)} \\
&=\frac{d}{d t} \lambda(t) \cdot \int_{M} f(t)^{2} d V_{g(t)}+\lambda(t) \int_{M} f(t) \frac{\partial}{\partial t} f(t) d V_{g(t)} .
\end{aligned}
$$

Since

$$
\begin{aligned}
-\int_{M} f(t) \Delta\left(\frac{\partial}{\partial t} f(t)\right) d V_{g(t)} & =-\int_{M} \Delta_{g(t)} f(t) \cdot \frac{\partial}{\partial t} f(t) d V_{g(t)} \\
& =\lambda(t) \int_{M} f(t) \frac{\partial}{\partial t} f(t) d V_{g(t)}
\end{aligned}
$$

it follows that

$$
\frac{d}{d t} \lambda(t) \cdot \int_{M} f(t)^{2} d V_{g(t)}=-\int_{M} f(t)\left(\frac{\partial}{\partial t} \Delta_{g(t)}\right) f(t) d V_{g(t)} .
$$

If we set $v_{i j}=-2 R_{i j}+4 \partial_{i} u \partial_{j} u$,

$$
\frac{\partial}{\partial t} \Gamma_{i j}^{k}=\frac{1}{2} g^{k \ell}\left(\partial_{i} v_{\ell j}+\partial_{j} v_{i l}-\partial_{\ell} v_{i j}\right)
$$

We temporarily omit all subscripts $t$. Multiplying with $g^{i j}$ on both sides, we obtain

$$
\begin{aligned}
g^{i j} \frac{\partial}{\partial t} \Gamma_{i j}^{k} & =\frac{1}{2} g^{k l}\left(2 \nabla^{i} v_{l i}-\nabla_{l}\left(g^{i j} v_{i j}\right)\right)=g^{k l} \nabla^{i} v_{i l}+\nabla^{k} S \\
& =g^{k l} \nabla^{i}\left(-2 R_{i l}+4 \nabla_{i} u \nabla_{l} u\right)+\nabla^{k}\left(R-2|\nabla u|^{2}\right)
\end{aligned}
$$




$$
\begin{aligned}
& =-\nabla^{k} R+4 \Delta u \cdot \nabla^{k} u+4 \nabla_{i} u \cdot \nabla^{i} \nabla^{k} u+\nabla^{k} R-4 \nabla^{k} \nabla^{i} u \cdot \nabla_{i} u \\
& =4 \Delta u \cdot \nabla^{k} u .
\end{aligned}
$$

Therefore,

$$
\begin{aligned}
\frac{\partial}{\partial t}(\Delta f) & =\frac{\partial}{\partial t}\left(g^{i j} \nabla_{i} \nabla_{j} f\right) \\
& =\left(\frac{\partial}{\partial t} g^{i j}\right) \nabla_{i} \nabla_{j} f+g^{i j}\left[\partial_{i} \partial_{j} \frac{\partial f}{\partial t}-\left(\frac{\partial}{\partial t} \Gamma_{i j}^{k}\right) \partial_{k} f-\Gamma_{i j}^{k} \partial_{k} \frac{\partial f}{\partial t}\right] \\
& =\left(\frac{\partial}{\partial t} g^{i j}\right) \nabla_{i} \nabla_{j} f+\Delta_{g(t)}\left(\frac{\partial}{\partial t} f\right)-g^{i j}\left(\frac{\partial}{\partial t} \Gamma_{i j}^{k}\right) \nabla_{k} f \\
& =\left(2 R_{i j}-4 \nabla_{i} u \nabla_{j} u\right) \nabla^{i} \nabla^{j} f-4 \Delta u \cdot \nabla^{k} u \nabla_{k} f+\Delta_{g(t)}\left(\frac{\partial}{\partial t} f\right) .
\end{aligned}
$$

Plugging this into (7-4), we derive

$$
\begin{aligned}
& \frac{d}{d t} \lambda(t) \cdot \int_{M} f(t)^{2} d V_{g(t)} \\
& \quad=-2 \int_{M} R_{i j} \nabla^{i} \nabla^{j} f d V+4 \int_{M} f \nabla^{i} u \nabla^{j} u \nabla_{i} \nabla_{j} f d V+4 \int_{M} f \Delta u \cdot \nabla^{k} u \nabla_{k} f d V .
\end{aligned}
$$

The first term can be rewritten as

$$
\begin{aligned}
-2 \int_{M} f R_{i j} \nabla^{i} \nabla^{j} f d V & =\int_{M} \nabla^{i}\left(2 f R_{i j}\right) \nabla^{j} f d V \\
& =2 \int_{M}\left(\nabla^{i} f \cdot R_{i j}+f \cdot \nabla^{i} R_{i j}\right) \nabla^{j} f d V \\
& =2 \int_{M} R_{i j} \nabla^{i} f \nabla^{j} f d V+\int_{M} f \nabla_{j} R \nabla^{j} f d V \\
& =2 \int_{M} R_{i j} \nabla^{i} f \nabla^{j} f d V-\int_{M} R \nabla_{j}\left(f \nabla^{j} f\right) d V \\
& =\lambda \int_{R} f^{2} d V-\int_{M} R|\nabla f|^{2} d V+2 \int_{M} R_{i j} \nabla^{i} f \nabla^{j} f d V .
\end{aligned}
$$

Hence

$$
\begin{aligned}
& \left(\frac{d}{d t} \lambda(t)\right) \int_{M} f(t)^{2} d V_{g(t)} \\
& =\lambda(t) \int_{M} R_{g(t)} f(t)^{2} d V_{g(t)}+2 \int_{M} R_{i j} \nabla^{i} f \nabla^{j} f d V-\int_{M} R_{g(t)}\left|\nabla_{g(t)} f(t)\right|_{g(t)}^{2} d V_{g(t)} \\
& \quad+4 \int_{M} f\left(\nabla^{i} u \nabla^{j} u \nabla_{i} \nabla_{j} f+\Delta u \nabla^{k} u \nabla_{k} f\right) d V .
\end{aligned}
$$


On the other hand,

$$
\begin{aligned}
& \int_{M} f \nabla^{i} u \nabla^{j} u \nabla_{i} \nabla_{j} f d V \\
& =-\int_{M} \nabla_{i}\left(f \nabla^{i} u \nabla^{j} u\right) \nabla_{j} f d V \\
& =-\int_{M}\left(\nabla_{i} f \nabla^{i} u \nabla^{j} u+f \Delta u \nabla^{j} u+f \nabla^{i} u \nabla_{i} \nabla^{j} u\right) \nabla_{j} f d V \\
& =-\int_{M} f \Delta u\langle\nabla u, \nabla f\rangle d V-\int_{M} \nabla^{i} u \nabla^{j} u \nabla_{i} f \nabla_{j} f d V-\int_{M} f \nabla^{i} u \nabla^{j} f \nabla_{i} \nabla_{j} u d V
\end{aligned}
$$

and therefore

$$
\begin{aligned}
\frac{d}{d t} \lambda(t) \int_{M} f(t)^{2} d V_{g(t)}= & \lambda(t) \int_{M} R_{g(t)} f(t)^{2} d V_{g(t)}-4 \int_{M} f \nabla^{i} u \nabla^{j} f \nabla_{i} \nabla_{j} u d V \\
& +2 \int_{M} S_{i j} \nabla^{i} f \nabla_{j} f d V-\int_{M} R_{g(t)}\left|\nabla_{g(t)} f(t)\right|_{g(t)}^{2} d V_{g(t)} .
\end{aligned}
$$

The last term here can be simplified as follows:

$$
\begin{aligned}
-\int_{M} f \nabla^{i} u & \nabla^{j} f \nabla_{i} \nabla_{j} u d V \\
& =\int_{M} \nabla^{j}\left(f \nabla_{i} u \nabla_{j} f\right) \nabla^{i} u d V \\
& =\int_{M}\left(\nabla^{j} f \nabla_{i} u \nabla_{j} f+f \nabla^{j} \nabla_{i} u \nabla_{j} f+f \nabla_{i} u \Delta f\right) \nabla^{i} u d V \\
& =\int_{M}|\nabla u|^{2}|\nabla f|^{2} d V+\int_{M} f \Delta f|\nabla u|^{2} d V+\int_{M} f \nabla^{i} u \nabla^{j} f \nabla_{i} \nabla_{j} u d V .
\end{aligned}
$$

Consequently,

$$
-2 \int_{M} f \nabla^{i} u \nabla^{j} f \nabla_{i} \nabla_{j} u d V=\int_{M}|\nabla u|^{2}|\nabla f|^{2} d V-\lambda \int_{M} f^{2}|\nabla u|^{2} d V .
$$

Therefore we derive the following.

Theorem 7.1. If $(g(t), u(t))$ is a solution of the harmonic-Ricci flow on a compact Riemannian manifold $M$ and $\lambda(t)$ denotes the eigenvalue of the Laplacian $\Delta_{g(t)}$, then

$$
\begin{aligned}
\frac{d}{d t} \lambda(t) \cdot \int_{M} f(t)^{2} d V_{g(t)} & \\
=\lambda(t) \int_{M} S_{g(t), u(t)} f(t)^{2} d V_{g(t)} & -\int_{M} S_{g(t), u(t)}\left|\nabla_{g(t)} f(t)\right|_{g(t)}^{2} d V_{g(t)} \\
& +2 \int_{M}\left\langle\varphi_{g(t), u(t)}, d f(t) \otimes d f(t)\right\rangle d V_{g(t)} .
\end{aligned}
$$


We set

$$
S_{\min }(0):=\min _{x \in M} S(x, 0)
$$

Theorem 7.2. Let $(g(t), u(t))_{t \in[0, T]}$ be a solution of the harmonic-Ricci flow on a compact Riemannian manifold $M$ and let $\lambda(t)$ denote the eigenvalue of the Laplacian $\Delta_{g(t)}$. Suppose that $\mathscr{S}_{g(t), u(t)}-\alpha S_{g(t), u(t)} g(t) \geq 0$ along the harmonic-Ricci flow for some $\alpha \geq \frac{1}{2}$.

(1) If $S_{\min }(0) \geq 0, \lambda(t)$ is nondecreasing along the harmonic-Ricci flow for any $t \in[0, T]$.

(2) If $S_{\min }(0)>0$, the quantity

$$
\left(1-\frac{2}{n} S_{\min }(0) t\right)^{n \alpha} \lambda(t)
$$

is nondecreasing along the harmonic-Ricci flow for $T \leq n /\left(2 S_{\min }(0)\right)$.

(3) If $S_{\min }(0)<0$, the quantity

$$
\left(1-\frac{2}{n} S_{\min }(0) t\right)^{n \alpha} \lambda(t)
$$

is nondecreasing along the harmonic-Ricci flow for any $t \in[0, T]$.

Proof. By Theorem 7.1, we have

$$
\frac{d}{d t} \lambda(t) \geq \frac{\int_{M} S_{g(t), u(t)} f(t)^{2} d V_{g(t)}}{\int_{M} f(t)^{2} d V_{g(t)}} \lambda(t)+(2 \alpha-1) \frac{\int_{M} S_{g(t), u(t)}\left|\nabla_{g(t)} f(t)\right|_{g(t)}^{2}}{\int_{M} f(t)^{2} d V_{g(t)}} .
$$

By definition we have $-f(t) \Delta_{g(t)}=\lambda(t) f(t)$. Integrating both sides yields that $\lambda(t) \geq 0$. Since

$$
\frac{\partial}{\partial t} S_{g(t), u(t)}=\Delta_{g(t)} S_{g(t), u(t)}+2\left|\mathscr{Y}_{g(t), u(t)}\right|_{g(t)}^{2}+4\left|\Delta_{g(t)} u(t)\right|_{g(t)}^{2}
$$

and $\left|\mathscr{S}_{g(t), u(t)}\right|^{2} \geq(1 / n) S_{g(t), u(t)}^{2}$, it follows that

$$
\frac{\partial}{\partial t} S_{g(t), u(t)} \geq \Delta_{g(t)} S_{g(t), u(t)}+\frac{2}{n} S_{g(t), u(t)}^{2} .
$$

The corresponding ODE

$$
\frac{d}{d t} a(t)=\frac{2}{n} a(t)^{2}, \quad a(t)=S_{\min }(0)
$$

has the solution

$$
a(t)=\frac{S_{\min }(0)}{1-(2 / n) S_{\min }(0) t} .
$$


Then the maximum principle implies $S_{g(t), u(t)} \geq a(t)$ and hence, using the assumption that $2 \alpha-1 \geq 0$,

$$
\frac{d}{d t} \lambda(t) \geq a(t) \lambda(t)+(2 \alpha-1) a(t) \frac{\int_{M}\left|\nabla_{g(t)} f(t)\right|_{g(t)}^{2} d V_{g(t)}}{\int_{M} f(t)^{2} d V_{g(t)}} .
$$

By integration by parts, we note that

$$
\int_{M}|\nabla f|^{2} d V=-\int_{M} f \cdot \Delta f d V=\lambda \int_{M} f^{2} d V,
$$

which shows that

$$
\frac{d}{d t} \lambda(t) \geq a(t) \lambda(t)+(2 \alpha-1) a(t) \lambda=2 \alpha a(t) \lambda(t)
$$

and

$$
\frac{d}{d t}\left(\lambda(t) \cdot \exp \left(-2 \alpha \int_{0}^{t} a(\tau) d \tau\right)\right) \geq 0 .
$$

This inequality clearly implies the desired result. If $S_{\min }(0) \geq 0$, by the nonnegativity of $\mathscr{Y}_{g(t)}$ preserved along the harmonic-Ricci flow, we conclude that $d \lambda(t) / d t \geq 0$.

Corollary 7.3. Let $(g(t), u(t))_{t \in[0, T]}$ be a solution of the harmonic-Ricci flow on a compact Riemannian surface $\Sigma$ and let $\lambda(t)$ denote the eigenvalue of the Laplacian $\Delta_{g(t)}$.

(1) Suppose that $\operatorname{Ric}_{g(t)} \leq \epsilon d u(t) \otimes d u(t)$ where

$$
\epsilon \leq 4 \frac{1-\alpha}{1-2 \alpha}, \quad \alpha>\frac{1}{2} .
$$

(i) If $S_{\min }(0) \geq 0, \lambda(t)$ is nondecreasing along the harmonic-Ricci flow for any $t \in[0, T]$.

(ii) If $S_{\min }(0)>0$, the quantity

$$
\left(1-S_{\min }(0) t\right)^{2 \alpha} \lambda(t)
$$

is nondecreasing along the harmonic-Ricci flow for $T \leq 1 / S_{\min }(0)$.

(iii) If $S_{\min }(0)<0$, the quantity

$$
\left(1-S_{\min }(0) t\right)^{2 \alpha} \lambda(t)
$$

is nondecreasing along the harmonic-Ricci flow for any $t \in[0, T]$.

(2) Suppose that

$$
\left|\nabla_{g(t)} u(t)\right|_{g(t)}^{2} g(t) \geq 2 d u(t) \otimes d u(t) .
$$

(i) If $S_{\min }(0) \geq 0, \lambda(t)$ is nondecreasing along the harmonic-Ricci flow for any $t \in[0, T]$. 
(ii) If $S_{\min }(0)>0$, the quantity

$$
\left(1-S_{\min }(0) t\right) \lambda(t)
$$

is nondecreasing along the harmonic-Ricci flow for $T \leq 1 / S_{\min }(0)$.

(iii) If $S_{\min }(0)<0$, the quantity

$$
\left(1-S_{\min }(0) t\right) \lambda(t)
$$

is nondecreasing along the harmonic-Ricci flow for any $t \in[0, T]$.

Proof. As above, we always omit subscripts $t$. In the surface case, we have $R_{i j}=\frac{1}{2} R g_{i j}$. Then

$$
\begin{aligned}
T_{i j} & :=S_{i j}-\alpha S g_{i j}=\frac{R}{2} g_{i j}-2 \nabla_{i} u \nabla_{j} u-\alpha\left(R-2|\nabla u|^{2}\right) g_{i j} \\
& =\left(\frac{1}{2}-\alpha\right) R g_{i j}-2 \nabla_{i} u \nabla_{j} u+2 \alpha|\nabla u|^{2} g_{i j} .
\end{aligned}
$$

For any vector $V=\left(V^{i}\right)$, we calculate

$$
\begin{aligned}
T_{i j} V^{i} V^{j} & =\left(\frac{1}{2}-\alpha\right) R|V|^{2}-2\left(\nabla_{i} u V^{i}\right)^{2}+2 \alpha|\nabla u|^{2}|V|^{2} \\
& \geq\left(\frac{1}{2}-\alpha\right) R|V|^{2}-2|\nabla u|^{2}|V|^{2}+2 \alpha|\nabla u|^{2}|V|^{2} .
\end{aligned}
$$

If $R_{i j} \leq \epsilon \nabla_{i} u \nabla_{j} u$, then $T_{i j} V^{i} V^{j}=\left[\left(\frac{1}{2}-\alpha\right) \epsilon-2+2 \alpha\right]|\nabla u|^{2}|V|^{2} \geq 0$.

For the second case, we note that

$$
\begin{aligned}
T_{i j} V^{i} V^{j} & =R_{i j} V^{i} V^{j}-2 \nabla_{i} u V^{i} \nabla_{j} u V^{j}-\frac{R}{2}|V|^{2}+|\nabla u|^{2}|V|^{2} \\
& \geq R_{i j} V^{i} V^{j}-|\nabla u|^{2}|V|^{2}-\frac{R}{2}|V|^{2}+|\nabla u|^{2}|V|^{2}=0 .
\end{aligned}
$$

Hence the corresponding results follow by Theorem 7.2.

When we consider the Ricci flow, we have the following two results derived from Corollary 7.3.

Corollary 7.4. Let $(g(t))_{t \in[0, T]}$ be a solution of the Ricci flow on a compact Riemannian surface $\Sigma$ and let $\lambda(t)$ denote the eigenvalue of the Laplacian $\Delta_{g(t)}$.

(1) If $R_{\min }(0) \geq 0, \lambda(t)$ is nondecreasing along the Ricci flow for any $t \in[0, T]$.

(2) If $R_{\min }(0)>0$, the quantity $\left(1-R_{\min }(0) t\right) \lambda(t)$ is nondecreasing along the Ricci flow for $T \leq 1 / R_{\min }(0)$.

(3) If $R_{\min }(0)<0$, the quantity $\left(1-R_{\min }(0) t\right) \lambda(t)$ is nondecreasing along the Ricci flow for any $t \in[0, T]$. 
Remark 7.5. Let $(g(t))_{t \in[0, T]}$ be a solution of the Ricci flow on a compact Riemannian surface $\Sigma$ with nonnegative scalar curvature and let $\lambda(t)$ denote the eigenvalue of the Laplacian $\Delta_{g(t)}$. Then $\lambda(t)$ is nondecreasing along the Ricci flow for $t \in[0, T]$.

\section{Eigenvalues of the Laplacian-type under the harmonic-Ricci flow}

Recall that

$$
\mu(g, u)=\mu_{1}(g, u)=\inf \left\{\mathscr{F}(g, u, f) \mid \int_{M} e^{-f} d V_{g}=1\right\} .
$$

We showed that $\mu(g, u)$ is the smallest eigenvalue of the operator

$$
-4 \Delta_{g}+R_{g}-2\left|\nabla_{g} u\right|_{g}^{2}
$$

Inspired by [Cao 2007; 2008], we define a Laplacian-type operators associated with quantities $g, u, c$ :

$$
\begin{aligned}
\Delta_{g, u, c} & :=-\Delta_{g}+c\left(R_{g}-2\left|\nabla_{g} u\right|_{g}^{2}\right), \\
\Delta_{g, u} & :=\Delta_{g, u, \frac{1}{2}}=-\Delta_{g}+\frac{1}{2}\left(R_{g}-2\left|\nabla_{g} u\right|_{g}^{2}\right) .
\end{aligned}
$$

Then $\mu(g, u)$ is the smallest eigenvalue of the operator $4 \Delta_{g, u, 1 / 4}$.

To the operator $\Delta_{g, u}$ we associate the functional

$$
C^{\infty}(M) \rightarrow \mathbb{R}, \quad f \mapsto \lambda_{g, u}(f):=\int_{M} f \Delta_{g, u} f d V_{g}
$$

When $f$ is an eigenfunction of the operator $\Delta_{g, u}$ with the eigenvalue $\lambda$, that is, $\Delta_{g, u} f=\lambda f$ and is normalized by $\int_{M} f^{2} d V_{g}=1$, we obtain $\lambda_{g, u}(f)=\lambda$. The next lemma will deal with the evolution equation for $\lambda(f(t))$, where $f(t)$ is an eigenfunction of $\Delta_{g(t), u(t)}$ and the couple $(g(t), u(t))$ satisfies the harmonic-Ricci flow. Set

$$
v_{i j}:=-2 S_{i j}=-2 R_{i j}+4 \partial_{i} u \cdot \partial_{j} u, \quad v:=g^{i j} v_{i j} .
$$

The symmetric tensor field thus obtained is denoted by $\mathscr{V}_{g(t), u(t)}=\left(v_{i j}\right)$.

Lemma 8.1. Suppose that $(g(t), u(t))$ is a solution of the harmonic-Ricci flow on a compact Riemannian manifold $M$ and $f(t)$ is an eigenfunction of $\Delta_{g(t), u(t)}$, that is, $\Delta_{g(t), u(t)} f(t)=\lambda(t) f(t)$ (where $\lambda(t)$ is only a function of time $t$ only), with the normalized condition

$$
\int_{M} f(t)^{2} d V_{g(t)}=1
$$

Then we have 
(8-6) $\frac{d}{d t} \lambda_{g(t), u(t)}(f(t)$

$$
\begin{aligned}
=\int_{M} f(t) & \left(\nabla^{i} v_{i k}-\frac{1}{2} \nabla_{k} v\right) \nabla^{k} f(t) d V_{g(t)}-\int_{M} f^{2}(t) \frac{\partial}{\partial t}\left|\nabla_{g(t)} u(t)\right|_{g(t)}^{2} d V_{g(t)} \\
+ & \int_{M}\left(\left\langle V_{g(t), u(t)}, \nabla_{g(t)}^{2} f(t)\right\rangle_{g(t)}+\frac{1}{2}\left(\frac{\partial}{\partial t} R_{g(t)}\right) f(t)\right) f(t) d V_{g(t)} .
\end{aligned}
$$

Before proving the lemma, we recall a formula that is an immediate consequence of the evolution equation:

$$
\begin{aligned}
& \frac{\partial}{\partial t}\left(\Delta_{g(t)} f\right) \\
& \quad=-g^{i p} g^{j q} v_{p q} \nabla_{i} \nabla_{j} f-g^{i j} g^{k \ell} \nabla_{i} v_{j \ell} \nabla_{k} f+\frac{1}{2}\left\langle\nabla_{g(t)} v_{g(t)}, \nabla_{g(t)} f(t)\right\rangle_{g(t)}
\end{aligned}
$$

where the metric $g(t)$ evolves by $\partial g_{i j} / \partial t=v_{i j}$.

Proof. Using (8-7) and integration by parts, we get

$$
\begin{aligned}
& \frac{d}{d t} \lambda_{g(t), u(t)}(f(t)) \\
& =\frac{\partial}{\partial t} \int_{M}\left(-\Delta_{g(t)} f(t)+\left(\frac{R_{g(t)}}{2}-\left|\nabla_{g(t)} u(t)\right|_{g(t)}^{2}\right) f(t)\right) f(t) d V_{g(t)} \\
& =\int_{M}\left(g^{i p} g^{j q} v_{p q} \nabla_{i} \nabla_{j} f+g^{i j} g^{k l} \nabla_{i} v_{j l} \nabla_{k} f-\frac{1}{2}\left\langle\nabla_{g(t)} v_{g(t)}, \nabla_{g(t)} f(t)\right\rangle_{g(t)}\right) f(t) d V_{g(t)} \\
& \quad+\int_{M}\left(-\Delta_{g(t)}\left(\frac{\partial}{\partial t} f(t)\right)+\left(\frac{R_{g(t)}}{2}-\left|\nabla_{g(t)} u(t)\right|_{g(t)}^{2}\right) \frac{\partial}{\partial t} f(t)\right. \\
& \left.\quad+\left(\frac{\partial}{\partial t}\left(\frac{1}{2} R_{g(t)}\right)-\frac{\partial}{\partial t}\left(\left|\nabla_{g(t)} u(t)\right|_{g(t)}^{2}\right)\right) f(t)\right) f(t) d V_{g(t)} \\
& \quad+\int_{M}\left(-\Delta_{g(t)} f(t)+\left(\frac{R_{g(t)}}{2}-\left|\nabla_{g(t)} u\right|_{g(t)}^{2}\right) f(t)\right) \frac{\partial}{\partial t}\left(f(t) d V_{g(t)}\right) \\
& =\int_{M}\left(g^{i p} g^{j q} v_{p q} \nabla_{i} \nabla_{j} f+\frac{1}{2}\left(\frac{\partial}{\partial t} R_{g(t)}\right) f(t)\right) f(t) d V_{g(t)} \\
& \quad+\int_{M}\left(g^{i j} g^{k l} \nabla_{i} v_{j l} \nabla_{k} f-\frac{1}{2} g^{k l} \nabla_{l} v \nabla_{k} f\right) f(t) d V_{g(t)} \\
& \quad+\int_{M} \Delta_{g(t), u(t)} f(t)\left(\frac{\partial}{\partial t} f(t) d V_{g(t)}+\frac{\partial}{\partial t}\left(f(t) d V_{g(t)}\right)\right) \\
& \quad-\int \frac{\partial}{\partial t}\left(\left|\nabla_{g(t)} u(t)\right|_{g(t)}^{2}\right) f(t)^{2} d V_{g(t)} .
\end{aligned}
$$

Since $f(t)$ is an eigenfunction of $\Delta_{g(t), u(t)}$, it follows that

$$
\int_{M} \Delta_{g(t), u(t)} f(t)\left(\frac{\partial}{\partial t} f(t) d V_{g(t)}+\frac{\partial}{\partial t}\left(f(t) d V_{g(t)}\right)\right)=\lambda(t) \frac{\partial}{\partial t} \int_{M} f(t)^{2} d V_{g(t)}=0
$$

by the normalization condition. This completes the proof. 
Using (3-6), we find that the first term in the right side of (8-6) can be written as

$$
\begin{gathered}
\int_{M}\left(v_{i j} \nabla^{i} \nabla^{j} f+\frac{1}{2}\left(\frac{\partial}{\partial t} R_{g(t)}\right) f(t)\right) f(t) d V_{g(t)} \\
=\int_{M}\left(-2 f(t)\left\langle\operatorname{Ric}_{g(t)}, \nabla_{g(t)}^{2} f(t)\right\rangle_{g(t)}+4 f(t)\left\langle d u(t) \otimes d u(t), \nabla_{g(t)}^{2} f(t)\right\rangle_{g(t)}\right) d V_{g(t)} \\
\quad+\int_{M}\left(\left(\frac{1}{2} \Delta_{g(t)} R_{g(t)}+\left|\operatorname{Ric}_{g(t)}\right|_{g(t)}^{2}\right) f(t)^{2}+2 f(t)^{2}\left|\Delta_{g(t)} u(t)\right|_{g(t)}^{2}\right. \\
\left.\quad-2 f(t)^{2}\left|\nabla_{g(t)}^{2} u(t)\right|_{g(t)}^{2}-4 f(t)^{2}\left\langle\operatorname{Ric}_{g(t)}, d u(t) \otimes d u(t)\right\rangle_{g(t)}\right) d V_{g(t)} \\
=\int_{M}\left(-2 f(t)\left\langle\operatorname{Ric}_{g(t)}, \nabla_{g(t)}^{2} f(t)\right\rangle_{g(t)}+\left(\frac{1}{2} \Delta_{g(t)} R_{g(t)}+\left|\operatorname{Ric}_{g(t)}\right|_{g(t)}^{2}\right) f(t)^{2}\right) d V_{g(t)} \\
\quad+\int_{M}\left(4 f(t)\left\langle d u \otimes d u, \nabla_{g(t)}^{2} f(t)\right\rangle_{g(t)}-4 f^{2}\left\langle d u(t) \otimes d u(t), \operatorname{Ric}_{g(t)}\right\rangle_{g(t)}\right. \\
\left.+2 f(t)^{2}\left|\Delta_{g(t)} u(t)\right|_{g(t)}^{2}-2 f(t)^{2}\left|\nabla_{g(t)}^{2} u(t)\right|_{g(t)}^{2}\right) d V_{g(t)}
\end{gathered}
$$

For the second term in (8-6), using the contracted Bianchi identities, one has

$$
\begin{gathered}
\int_{M}\left(g^{i j} \nabla_{i} v_{j k}-\frac{1}{2} \nabla_{k} v\right) \nabla^{k} f \cdot f(t) d V_{g(t)} \\
=\int_{M}\left(g^{i j} \nabla_{i}\left(-2 R_{j k}+4 \partial_{j} u \partial_{k} u\right)\right. \\
\left.\quad-\frac{1}{2} \nabla_{k}\left(-2 R_{g(t)}+4\left|\nabla_{g(t)} u(t)\right|_{g(t)}^{2}\right)\right) \nabla^{k} f \cdot f(t) d V_{g(t)} \\
=\int_{M} 4 f(t) \Delta_{g(t)} u(t)\left\langle\nabla_{g(t)} u(t), \nabla_{g(t)} f(t)\right\rangle_{g(t)} d V_{g(t)} \\
\quad+\int_{M}\left(4 g^{i j} \nabla_{j} u \cdot \nabla_{i} \nabla_{k} u-2 \nabla_{k}\left|\nabla_{g(t)} u(t)\right|_{g(t)}^{2}\right) \nabla^{k} f \cdot f(t) d V_{g(t)} \\
=\int_{M} 4 f(t) \Delta_{g(t)} u(t)\left\langle\nabla_{g(t)} u(t), \nabla_{g(t)} f(t)\right\rangle_{g(t)} d V_{g(t)}
\end{gathered}
$$

where in the last step we use the identity $\nabla_{k}|\nabla u|^{2}=2 g^{p q} \nabla_{k} \nabla_{p} u \cdot \nabla_{q} u$. Therefore

$$
\begin{aligned}
& \text { (8-8) } \frac{d}{d t} \lambda_{g(t), u(t)}(f(t)) \\
& \begin{array}{r}
=\int_{M}\left(-2 f(t)\left\langle\operatorname{Ric}_{g(t)}, \nabla_{g(t)}^{2} f(t)\right\rangle_{g(t)}+\left(\frac{1}{2} \Delta_{g(t)} R_{g(t)}+\left|\operatorname{Ric}_{g(t)}\right|_{g(t)}^{2}\right) f(t)^{2}\right) d V_{g(t)} \\
+4 f(t) \int_{M}\left(\left\langle d u(t) \otimes d u(t), \nabla_{g(t)}^{2} f(t)\right\rangle_{g(t)}-f(t)\left\langle d u(t) \otimes d u(t), \operatorname{Ric}_{g(t)}\right\rangle_{g(t)}\right. \\
+2 f(t)^{2}\left|\Delta_{g(u)} u(t)\right|_{g(t)}^{2}-2 f(t)^{2}\left|\nabla_{g(t)}^{2} u(t)\right|_{g(t)}^{2} \\
\left.\quad+4 f(t) \Delta_{g(t)} u(t)\left\langle\nabla_{g(t)} u(t), \nabla_{g(t)} f(t)\right\rangle_{g(t)}\right) d V_{g(t)} \\
-\int_{M}\left(\Delta_{g(t)}\left|\nabla_{g(t)} u(t)\right|_{g(t)}^{2}-2\left|\nabla_{g(t)}^{2} u(t)\right|_{g(t)}^{2}-4\left|\nabla_{g(t)} u(t)\right|_{g(t)}^{4}\right) f(t)^{2} d V_{g(t)} .
\end{array}
\end{aligned}
$$


The above evolution equation can be simplified as follows.

Theorem 8.2. Suppose $(g(t), u(t))$ is a solution of the harmonic-Ricci flow on a compact Riemannian manifold $M$ and $f(t)$ is an eigenfunction of $\Delta_{g(t), u(t)}$, that is, $\Delta_{g(t), u(t)} f(t)=\lambda(t) f(t)$ (where $\lambda(t)$ is only a function of time $t$ only), with the normalized condition $\int_{M} f(t)^{2} d V_{g(t)}=1$. Then we have

$$
\begin{aligned}
\frac{d}{d t} \lambda_{g(t), u(t)}(f(t))=\int_{M} 2\left\langle\mathscr{S}_{g(t)}, d f(t) \otimes d f(t)\right\rangle_{g(t)} d V_{g(t)} \\
\quad+\int_{M} f(t)^{2}\left(\left|\mathscr{S}_{g(t)}\right|_{g(t)}^{2}+2\left|\Delta_{g(t)} u(t)\right|_{g(t)}^{2}\right) d V_{g(t)} .
\end{aligned}
$$

Proof. Calculate

$$
\begin{aligned}
\int_{M} 4 f(t) & \Delta_{g(t)} u(t)\left\langle\nabla_{g(t)} u(t), \nabla_{g(t)} f(t)\right\rangle_{g(t)} d V_{g(t)} \\
= & -4 \int_{M} \nabla_{i} u\left[\nabla^{i} f \cdot\langle\nabla u, \nabla f\rangle+f\left(\nabla^{i}\langle\nabla u, \nabla f\rangle\right)\right] d V \\
= & -4 \int_{M}|\langle\nabla u, \nabla f\rangle|^{2} d V_{g}-4 \int_{M} f \nabla_{i} u\left(\left\langle\nabla^{i} \nabla u, \nabla f\right\rangle+\left\langle\nabla u, \nabla^{i} \nabla f\right) d V .\right.
\end{aligned}
$$

By the same method, we have

$$
\begin{aligned}
\int_{M}-\Delta_{g(t)}\left|\nabla_{g(t)} u(t)\right|_{g(t)}^{2} f(t)^{2} d & V_{g(t)} \\
& =-\int_{M}|\nabla u|^{2}\left(2 f \Delta f+2|\nabla f|^{2}\right) d V \\
& =-2 \int_{M}|\nabla f|^{2}|\nabla u|^{2} d V-2 \int_{M} f \Delta f|\nabla u|^{2} d V .
\end{aligned}
$$

However,

$$
\begin{aligned}
\int_{M} f \Delta f|\nabla u|^{2} d V & =\int_{M}-\nabla_{i} f \cdot \nabla^{i}\left(f|\nabla u|^{2}\right) d V \\
& =-\int_{M} \nabla_{i} f\left(\nabla^{i} f|\nabla u|^{2}+f \nabla^{i}|\nabla u|^{2}\right) d V \\
& =-\int_{M}|\nabla u|^{2}|\nabla f|^{2} d V-\int_{M} f \nabla_{i} f \cdot \nabla^{i}|\nabla u|^{2} d V .
\end{aligned}
$$

Therefore we arrive at

$$
\begin{aligned}
\int_{M}-\Delta_{g(t)}\left|\nabla_{g(t)} u(t)\right|_{g(t)}^{2} f(t)^{2} d & V_{g(t)} \\
& =2 \int_{M} f \nabla_{i} f \cdot \nabla^{i}|\nabla u|^{2} d V \\
& =4 \int_{M} f(t)\left\langle d u(t) \otimes d f(t), \nabla_{g(t)}^{2} u(t)\right\rangle_{g(t)} d V_{g(t)} .
\end{aligned}
$$


Using the contracted Bianchi identities, we may simplify the term $\int_{M} \frac{1}{2} f^{2} \Delta R d V$ as follows:

$$
\begin{aligned}
& \int_{N} \frac{f(t)^{2}}{2} \Delta_{g(t)} R_{g(t)} d V_{g(t)} \\
& =-\frac{1}{2} \int_{M} \nabla_{i} R \cdot \nabla^{i}\left(f^{2}\right) d V \\
& =-\int_{M} \nabla_{i} R \cdot f \nabla^{i} f d V=-2 \int_{M} \nabla^{k} R_{k i} \cdot f \nabla^{i} f d V \\
& =2 \int_{M} R_{k i} \nabla^{k}\left(f \nabla^{j} f\right) d V=2 \int_{M} R_{k i}\left(\nabla^{k} f \cdot \nabla^{j} f+f \nabla^{k} \nabla^{j} f\right) d V \\
& =2 \int_{M}\left\langle\operatorname{Ric}_{g(t)}, d f(t) \otimes d f(t)\right\rangle_{g(t)} d V_{g(t)}+2 \int_{M} f(t)\left\langle\operatorname{Ric}_{g(t)}, \nabla_{g(t)}^{2} f(t)\right\rangle_{g(t)} d V_{g(t)} .
\end{aligned}
$$

Hence (8-8) becomes

$$
\begin{aligned}
& \frac{d}{d t} \lambda_{g(t), u(t)}(f(t)) \\
& =\int_{M}\left(2\left\langle\operatorname{Ric}_{g(t)}, d f(t) \otimes d f(t)\right\rangle_{g(t)}+\left|\operatorname{Ric}_{g(t)}\right|_{g(t)}^{2} f(t)^{2}\right) d V_{g(t)} \\
& \quad+\int_{M}\left(2\left|\Delta_{g(t)} u(t)\right|_{g(t)}^{2}+4\left|\nabla_{g(t)} u(t)\right|_{g(t)}^{4}\right) f(t)^{2} d V_{g(t)} \\
& \quad-\int_{M} 4 f(t)^{2}\left\langle d u(t) \otimes d u(t), \operatorname{Ric}_{g(t)}\right\rangle_{g(t)} d V_{g(t)} \\
& \quad-\int_{M} 4\left|\left\langle\nabla_{g(t)} u(t), \nabla_{g(t)} f(t)\right\rangle_{g(t)}\right|^{2} d V_{g(t)} \\
& =\int_{M} 2\left\langle\varphi_{g(t)}, d f(t) \otimes d f(t)\right\rangle_{g(t)} d V_{g(t)} \\
& \quad+\int_{M} f(t)^{2}\left(\left|\operatorname{Ric}_{g(t)}-2 d u(t) \otimes d u(t)\right|_{g(t)}^{2}+2\left|\Delta_{g(t)} u(t)\right|_{g(t)}^{2}\right) d V_{g(t)}
\end{aligned}
$$

where, by definition, $S_{i j}=R_{i j}-2 \partial_{i} u \partial_{j} u$.

List [2006] proved that the nonnegativity of the operator $\mathscr{Y}_{g(t)}$ is preserved by the harmonic-Ricci flow. Hence we get the following.

Corollary 8.3. If $\operatorname{Ric}_{g(0)}-2 d u(0) \otimes d u(0) \geq 0$, the eigenvalues of the operator $\Delta_{g(t), u(t)}$ are nondecreasing under the harmonic-Ricci flow.

Remark 8.4. If we choose $u(t) \equiv 0$, we obtain Cao's result [2007]. 


\section{Another formula for $\frac{d}{d t} \lambda(f(t))$}

In this section we give another formula for $\frac{d}{d t} \lambda(f(t))$ using a method similar to that in [Li 2007]. Recall the formula

$$
\begin{aligned}
\frac{d}{d t} \lambda_{g(t), u(t)}(f(t))=\int_{M} 2\left\langle\mathscr{Y}_{g(t), u(t)}, d f(t) \otimes d f(t)\right\rangle_{g(t)} d V_{g(t)} \\
\quad+\int_{M} f(t)^{2}\left(\left|\mathscr{S}_{g(t), u(t)}\right|_{g(t)}^{2}+2\left|\Delta_{g(t)} u(t)\right|_{g(t)}^{2}\right) d V_{g(t)} .
\end{aligned}
$$

Consider the function $\varphi$ determined by $f^{2}(t)=e^{-\varphi(t)}$. Then we have

$$
d f=\frac{-e^{\varphi} d \varphi}{2 f}, \quad \frac{\nabla f}{f}=-\frac{\nabla \varphi}{2}, \quad \frac{\Delta f}{f}=-\frac{1}{2} \Delta \varphi+\frac{1}{4}|\nabla \varphi|^{2} .
$$

Hence

$2 \frac{d}{d t} \lambda_{g(t), u(t)}(f(t))=\int_{M}\left\langle\mathscr{Y}_{g(t), u(t)}, d \varphi(t) \otimes d \varphi(t)\right\rangle_{g(t)} e^{-\varphi(t)} d V_{g(t)}$

$$
+2 \int_{M}\left(\left|\varphi_{g(t), u(t)}\right|_{g(t)}^{2}+2\left|\Delta_{g(t)} u(t)\right|_{g(t)}^{2}\right) e^{-\varphi} d V_{g(t)} .
$$

Using integration by parts and contracted Bianchi identities yields

$$
\begin{aligned}
& \int_{M}\left\langle\mathscr{Y}_{g(t), u(t)}, d \varphi(t) \otimes d \varphi(t)\right\rangle_{g(t)} e^{-\varphi(t)} d V_{g(t)} \\
& =\int_{M} S_{i j} \nabla^{i} \varphi \nabla^{j} \varphi e^{-\varphi} d V=-\int_{M} S_{i j} \nabla^{j} \varphi \nabla^{i}\left(e^{-\varphi}\right) d V \\
& =\int_{M} e^{-\varphi} \nabla^{i}\left(S_{i j} \nabla^{j} \varphi\right) d V \\
& =\int_{M} \nabla^{i} S_{i j} \cdot \nabla^{j} \varphi \cdot e^{-\varphi} d V+\int_{M} S_{i j} \nabla^{i} \nabla^{j} \varphi \cdot e^{-\varphi} d V \\
& =\int_{M} \nabla^{i} R_{i j} \cdot \nabla^{j} \varphi \cdot e^{-\varphi} d V_{g}+\int_{M} S_{i j} \nabla^{i} \nabla^{j} \varphi \cdot e^{-\varphi} d V \\
& \quad+\int_{M} \nabla^{i}\left(-2 \nabla_{i} u \nabla_{j} u\right) \nabla^{j} \varphi \cdot e^{-\varphi} d V_{g} \\
& =\frac{1}{2} \int_{M} R \Delta\left(e^{-\varphi}\right) d V+\int_{M} S_{i j} \nabla^{i} \nabla^{j} \varphi \cdot e^{-\varphi} d V-2 \int_{M}\left(\nabla^{i} u \nabla_{j} u\right) \nabla^{i} \nabla^{j}\left(e^{-\varphi}\right) d V .
\end{aligned}
$$

Thus

$$
\begin{aligned}
\int_{M} S_{i j} & \nabla^{i} \nabla^{j} \varphi \cdot e^{-\varphi} d V \\
& =\int_{M} S_{i j} \nabla^{i} \varphi \nabla^{j} \varphi e^{-\varphi} d V-\frac{1}{2} \int_{M} R \Delta\left(e^{-\varphi}\right) d V+2 \int_{M}\left(\nabla^{i} u \nabla_{j} u\right) \nabla^{i} \nabla^{j}\left(e^{-\varphi}\right) .
\end{aligned}
$$


On the other hand, one gets

$$
\begin{aligned}
\int_{M}\left|\nabla_{g(t)}^{2} \varphi(t)\right|_{g(t)}^{2} e^{-\varphi(t)} d V_{g(t)}=\int_{M} \nabla_{i} \nabla_{j} \varphi \nabla^{i} \nabla_{j} \varphi \cdot e^{-\varphi} d V \\
=-\int_{M} \nabla_{j} \varphi \cdot \nabla_{i} \nabla^{i} \nabla^{j} \varphi \cdot e^{-\varphi} d V-\int_{M} \nabla_{j} \varphi \cdot \nabla^{i} \nabla^{j} \varphi \cdot \nabla_{i}\left(e^{-\varphi}\right) d V \\
=-\int_{M} \nabla_{j} \varphi \cdot \nabla_{i} \nabla^{j} \nabla^{i} \varphi \cdot e^{-\varphi} d V-\int_{M} \nabla_{j} \varphi \cdot \nabla^{i} \nabla^{j} \varphi \cdot \nabla_{i}\left(e^{-\varphi}\right) d V .
\end{aligned}
$$

Since

$$
\begin{aligned}
\int_{M} \nabla_{j} \varphi \cdot \nabla^{i} \nabla^{j} \varphi \cdot \nabla_{i}\left(e^{-\varphi}\right) d V & =-\int_{M} \nabla^{i}\left(\nabla_{j} \varphi \cdot \nabla_{i}\left(e^{-\varphi}\right)\right) \nabla^{j} \varphi d V \\
= & -\int_{M} \nabla^{j} \varphi \cdot \nabla^{i} \nabla_{j} \varphi \cdot \nabla_{i}\left(e^{-\varphi}\right) d V-\int_{M}|\nabla \varphi|^{2} \Delta\left(e^{-\varphi}\right) d V,
\end{aligned}
$$

which implies

$$
\int_{M} \nabla_{j} \varphi \cdot \nabla^{i} \nabla^{j} \varphi \cdot \nabla_{i}\left(e^{-\varphi}\right) d V=-\frac{1}{2} \int_{M}|\nabla \varphi|^{2} \Delta\left(e^{-\varphi}\right) d V,
$$

it follows that

$$
\int_{M}\left|\nabla^{2} \varphi\right|^{2} e^{-\varphi} d V=-\int_{M} \nabla_{j} \varphi \cdot \nabla_{i} \nabla^{j} \nabla^{i} \varphi \cdot e^{-\varphi} d V+\frac{1}{2} \int_{M}|\nabla \varphi|^{2} \Delta\left(e^{-\varphi}\right) d V .
$$

By the Ricci identity the term $\nabla^{i} \nabla^{j} \nabla^{i} \varphi$ equals

$$
\begin{aligned}
\nabla_{i} \nabla^{j} \nabla^{i} \varphi & =g^{j k} g^{i l} \nabla_{i} \nabla_{k} \nabla_{l} \varphi=g^{j k} g^{i l}\left(\nabla_{k} \nabla_{i} \nabla_{l} \varphi-R_{i k l}^{p} \nabla_{p} \varphi\right) \\
& =\nabla^{j} \nabla_{i} \nabla^{i} \varphi-g^{j k} g^{i l} R_{i k l p} \nabla^{p} \varphi \\
& =\nabla^{j} \Delta \varphi+g^{j k} g^{i l} R_{i k p l} \nabla^{p} \varphi=\nabla^{j} \Delta \varphi+g^{j k} R_{k p} \nabla^{p} \varphi .
\end{aligned}
$$

Hence

$$
\begin{aligned}
-\int_{M} & \nabla_{j} \varphi \cdot \nabla_{i} \nabla^{j} \nabla^{i} \varphi \cdot e^{-\varphi} d V \\
= & -\int_{M} \nabla_{i} \varphi \cdot \nabla^{j} \Delta \varphi \cdot e^{-\varphi} d V-\int_{M} R_{k p} \nabla^{k} \varphi \cdot \nabla^{p} \varphi e^{-\varphi} d V \\
= & \int_{M} \nabla^{j} \Delta \varphi \cdot \nabla_{j}\left(e^{-\varphi}\right)+\int_{M} R_{k p} \nabla^{k} \varphi \cdot \nabla^{p}\left(e^{-\varphi}\right) d V \\
= & -\int_{M} \Delta \varphi \cdot \Delta\left(e^{-\varphi}\right)-\int_{M} e^{-\varphi}\left(\nabla^{p} R_{k p} \cdot \nabla^{k} \varphi+R_{k p} \nabla^{p} \nabla^{k} \varphi\right) \\
= & -\int_{M} \Delta\left(e^{-\varphi}\right) \cdot \Delta \varphi d V+\frac{1}{2} \int_{M} \nabla_{k} R \cdot \nabla^{k}\left(e^{-\varphi}\right) d V-\int_{M} e^{-\varphi} R_{k p} \nabla^{k} \nabla^{p} \varphi d V \\
= & -\int_{M} \Delta\left(e^{-\varphi}\right)\left(\Delta \varphi+\frac{1}{2} R\right)-\int_{M} R_{k p} \nabla^{k} \nabla^{p} \varphi \cdot e^{-\varphi} d V .
\end{aligned}
$$


Putting those formulas together, we obtain

$$
\begin{aligned}
& \int_{M} 2 S_{i j} \nabla^{i} \nabla^{j} \varphi \cdot e^{-\varphi} d V+\int_{M}\left|\nabla^{2} \varphi\right|^{2} e^{-\varphi} d V \\
&= \int_{M} S_{i j} \nabla^{i} \nabla_{j} \varphi \cdot e^{-\varphi} d V+\int_{M}\left(-2 \nabla_{i} u \nabla_{j} u\right) \nabla^{i} \nabla^{j} \varphi \cdot e^{-\varphi} d V \\
& \quad-\int_{M} \Delta\left(e^{-\varphi}\right)\left(\Delta \varphi+\frac{R}{2}-\frac{1}{2}|\nabla \varphi|^{2}\right) d V \\
&=\int_{M} S_{i j} \nabla^{i} \varphi \nabla^{j} \varphi \cdot e^{-\varphi} d V-\int_{M} \Delta\left(e^{-\varphi}\right)\left(\Delta \varphi+R-\frac{1}{2}|\nabla \varphi|^{2}\right) d V \\
&+2 \int_{M}\left(\nabla_{i} u \nabla_{j} u \cdot \nabla^{i} \nabla^{j}\left(e^{-\varphi}\right)-\nabla_{i} u \nabla_{j} u \cdot \nabla^{i} \nabla^{j} \varphi \cdot e^{-\varphi}\right) d V .
\end{aligned}
$$

Since $f$ is an eigenfunction of $\lambda$, it induces

$$
\lambda=-\frac{\Delta f}{f}+\frac{R}{2}-|\nabla u|^{2}=\frac{1}{2} \Delta \varphi-\frac{1}{4}|\nabla \varphi|^{2}+\frac{R}{2}-|\nabla u|^{2},
$$

and therefore

$$
\begin{aligned}
& \int_{M} 2 S_{i j} \nabla^{i} \nabla^{j} \varphi \cdot e^{-\varphi} d V+\int_{M}\left|\nabla^{2} \varphi\right|^{2} e^{-\varphi} d V \\
&=\int_{M} S_{i j} \nabla^{i} \varphi \nabla^{j} \varphi \cdot e^{-\varphi} d V-2 \int_{M} \Delta\left(|\nabla u|^{2}\right) \cdot e^{-\varphi} d V \\
& \quad+2 \int_{M} \nabla_{i} u \nabla_{j}\left(\nabla^{i} \nabla^{j}\left(e^{-\varphi}\right)-\nabla^{i} \nabla^{j} \varphi \cdot e^{-\varphi}\right) d V .
\end{aligned}
$$

Plugging this into the expression of $\frac{d}{d t} \lambda(f(t))$ yields

$2 \frac{d}{d t} \lambda_{g(t), u(t)}(f(t))$

$$
\begin{gathered}
=\int_{M} S_{i j} \nabla^{i} \varphi \nabla^{j} \varphi \cdot e^{-\varphi} d V+\int_{M}|\mathscr{S}|^{2} e^{-\varphi} d V+\int_{M}|\mathscr{S}|^{2} e^{-\varphi} d V+4 \int_{M}|\Delta u|^{2} e^{-\varphi} d V \\
=\int_{M}\left|\mathscr{Y}_{g(t), u(t)}+\nabla_{g(t)}^{2} \varphi(t)\right|_{g(t)}^{2} e^{-\varphi(t)} d V_{g(t)}+\int_{M}\left|\mathscr{Y}_{g(t), u(t)}\right|_{g(t)}^{2} e^{-\varphi(t)} d V_{g(t)} \\
+4 \int_{M}\left|\Delta_{g(t)} u(t)\right|_{g(t)}^{2} e^{-\varphi(t)} d V_{g(t)}+2 \int_{M} \Delta_{g(t)}\left|\nabla_{g(t)} u(t)\right|_{g(t)}^{2} e^{-\varphi(t)} d V_{g(t)} \\
+2 \int_{M} \nabla_{i} u \nabla_{j} u\left(-\nabla^{i} \nabla^{j}\left(e^{-\varphi}\right)+\nabla^{i} \nabla^{j} \varphi \cdot e^{-\varphi}\right) d V
\end{gathered}
$$

Now define

$$
\begin{aligned}
I: & =\int_{M}\left(\nabla_{i} u \nabla_{j} u \cdot \nabla^{i} \nabla^{j} \varphi\right) e^{-\varphi} d V=-\int_{M} \nabla^{i}\left(\nabla_{i} u \nabla_{j} u \cdot e^{-\varphi}\right) \nabla^{j} \varphi d V \\
& =-\int_{M} \nabla^{j} \varphi\left(\Delta u \cdot \nabla_{j} u \cdot e^{-\varphi}+\nabla_{i} u \nabla^{i} \nabla_{j} u \cdot e^{-\varphi}-\nabla_{i} u \nabla_{j} u \nabla^{i} \varphi \cdot e^{-\varphi}\right) d V \\
& =-\int_{M} \nabla_{j} u \nabla^{j} \varphi \Delta u \cdot e^{-\varphi} d V-\int_{M} \nabla_{i} u \nabla^{j} \varphi \nabla^{i} \nabla_{j} u \cdot e^{-\varphi} d V+\int_{M}|\langle d u, d \varphi\rangle|^{2} e^{-\varphi} d V,
\end{aligned}
$$




$$
\begin{aligned}
I I: & =\int_{M} \nabla_{i} u \nabla_{j} u \nabla^{i} \nabla^{j}\left(e^{-\varphi}\right) d V=\int_{M} \nabla^{i} \nabla^{j}\left(\nabla_{i} u \nabla_{j} u\right) e^{-\varphi} d V \\
& =\int_{M} \nabla^{i}\left(\nabla^{j} \nabla_{i} u \cdot \nabla_{j} u+\nabla_{i} u \Delta u\right) e^{-\varphi} d V \\
& =\int_{M}\left(\Delta \nabla^{i} u \cdot \nabla_{i} u+\nabla^{i} \Delta u \cdot \nabla_{i} u+\left|\nabla^{2} u\right|^{2}+|\Delta u|^{2}\right) e^{-\varphi} d V, \\
I I I: & =\int_{M} \Delta\left(|\nabla u|^{2}\right) e^{-\varphi} d V=2 \int_{M} \nabla^{i}\left(\nabla_{i} \nabla_{j} u \cdot \nabla^{j} u\right) e^{-\varphi} d V \\
& =2 \int_{M}\left(\Delta \nabla_{j} u \cdot \nabla^{j} u+\left|\nabla^{2} u\right|^{2}\right) e^{-\varphi} d V .
\end{aligned}
$$

If we set

$$
B:=2(I I I+I-I I),
$$

then

$$
\begin{aligned}
& \frac{B}{2}=\int_{M}\left(\Delta \nabla_{i} u \cdot \nabla^{i} u-\nabla_{i} \Delta u \cdot \nabla^{i} u+\left|\nabla^{2} u\right|^{2}-|\Delta u|^{2}+|\langle d u, d \varphi\rangle|^{2}\right. \\
& \left.-\nabla_{i} u \cdot \nabla^{i} \varphi \cdot \Delta u-\nabla_{i} u \cdot \nabla^{j} \varphi \cdot \nabla^{i} \nabla_{j} u\right) e^{-\varphi} d V \\
& =\int_{M}\left(R_{i j} \nabla^{i} u \nabla^{j} u+\left|\nabla^{2} u\right|^{2}-|\Delta u|^{2}+|\langle d u, d \varphi\rangle|^{2}\right. \\
& \left.-\nabla_{i} u \cdot \nabla^{i} \varphi \cdot \Delta u-\nabla_{i} u \cdot \nabla^{j} \varphi \cdot \nabla^{i} \nabla_{j} u\right) e^{-\varphi} d V .
\end{aligned}
$$

On the other hand,

$$
\begin{aligned}
-\int_{M} \nabla_{i} u \cdot \nabla^{i} \varphi \cdot \Delta u \cdot e^{-\varphi} d V & =\int_{M}\left(\nabla_{i} u \cdot \Delta u\right) \nabla^{i}\left(e^{-\varphi}\right) d V \\
& =-\int_{M} \nabla^{i}\left(\nabla_{i} u \cdot \Delta u\right) e^{-\varphi} d V \\
& =\int_{M}\left(-|\Delta u|^{2}-\nabla_{i} u \cdot \nabla^{i} \Delta u\right) e^{-\varphi} d V
\end{aligned}
$$

and

$$
\begin{aligned}
-\int_{M} \nabla_{i} u \nabla^{j} \varphi \nabla^{i} \nabla_{j} u \cdot e^{-\varphi} d V & =\int_{M} \nabla_{i} u \nabla^{i} \nabla_{j} u \nabla^{j}\left(e^{-\varphi}\right) d V \\
& =-\int_{M} \nabla^{j}\left(\nabla_{i} u \nabla^{i} \nabla_{j} u\right) e^{-\varphi} d V \\
& =\int_{M}\left(-\left|\nabla^{2} u\right|^{2}-\nabla_{i} u \Delta \nabla^{i} u\right) e^{-\varphi} d V .
\end{aligned}
$$

Therefore

$$
\frac{B}{2}=\int_{M}\left(-2|\Delta u|^{2}+|\langle d u, d \varphi\rangle|^{2}-2\langle\nabla u, \nabla \Delta u\rangle\right) e^{-\varphi} d V .
$$


By definition,

$$
\Delta\left(|\nabla u|^{2}\right)=\Delta\left(\nabla^{i} u \cdot \nabla_{i} u\right)=2 \nabla^{i} u \cdot \Delta \nabla_{i} u+2\left|\nabla^{2} u\right|^{2} .
$$

So

$$
\begin{aligned}
\Delta|\nabla u|^{2} & =2\left|\nabla^{2} u\right|^{2}+2\left(\nabla_{i} \Delta u+R_{i j} \nabla^{j} u\right) \nabla^{i} u \\
& =2\left|\nabla^{2} u\right|^{2}+2 R_{i j} \nabla^{i} u \cdot \nabla^{j} u+2\langle\nabla u, \nabla \Delta u\rangle .
\end{aligned}
$$

Plugging this into (9-1) yields

$$
\frac{B}{2}=\int_{M}\left(-2|\Delta u|^{2}+|\langle d u, d \varphi\rangle|^{2}+2\left|\nabla^{2} u\right|^{2}-\Delta|\nabla u|^{2}+2 R_{i j} \nabla^{i} u \nabla^{j} u\right) e^{-\varphi} d V .
$$

Since

$$
\begin{aligned}
2 R_{i j} \nabla^{i} u \nabla^{j} u & =2\left(S_{i j}+2 \nabla_{i} u \nabla_{j} u\right) \nabla^{i} u \nabla^{j} u \\
& =2 S_{i j} \nabla^{i} u \nabla^{j} u+4|\nabla u|^{4} \\
& =\frac{1}{4}|\mathscr{S}+4 d u \otimes d u|^{2}-\frac{1}{4}|\mathscr{Y}|^{2},
\end{aligned}
$$

it follows that

$$
\begin{aligned}
\frac{B}{2} & =I I I+I-I I \\
& =\int_{M}\left(|\langle d u, d \varphi\rangle|^{2}-2|\Delta u|^{2}-\frac{1}{4}|\mathscr{S}|^{2}+2\left|\nabla^{2} u\right|^{2}+\frac{1}{4}|\mathscr{S}+4 d u \otimes d u|^{2}\right) e^{-\varphi} d V-I I I .
\end{aligned}
$$

Hence

$$
B=\int_{M}\left(-4|\Delta u|^{2}+2|\langle d u, d \varphi\rangle|^{2}-\frac{1}{2}|\mathscr{9}|^{2}+4\left|\nabla^{2} u\right|^{2}+\frac{1}{2}|\mathscr{T}+4 d u \otimes d u|^{2}\right) e^{-\varphi} d V-2 I I I .
$$

Theorem 9.1. Suppose that $(g(t), u(t))$ is a solution of the harmonic-Ricci flow on a compact Riemannian manifold $M$ and $f(t)$ is an eigenfunction of $\Delta_{g(t), u(t)}$, that is, $\Delta_{g(t), u(t)} f(t)=\lambda(t) f(t)$ (where $\lambda(t)$ is only a function of time $t$ ), with the normalized condition $\int_{M} f(t)^{2} d V_{g(t)}=1$. Then we have

$$
\begin{aligned}
& \frac{d}{d t} \lambda(t) \\
& =\frac{d}{d t} \lambda_{g(t), u(t)}(f(t)) \\
& =\frac{1}{2} \int_{M}\left|\mathscr{\varphi}_{g(t), u(t)}+\nabla_{g(t)}^{2} \varphi(t)\right|_{g(t)}^{2} e^{-\varphi(t)} d V_{g(t)}+\frac{1}{4} \int_{M}\left|\mathscr{S}_{g(t), u(t)}\right|_{g(t)}^{2} e^{-\varphi(t)} d V_{g(t)} \\
& \quad+\int_{M}\left|\langle d u(t), d \varphi(t)\rangle_{g(t)}\right|^{2} e^{-\varphi(t)} d V_{g(t)}+2 \int_{M}\left|\nabla_{g(t)}^{2} u(t)\right|_{g(t)}^{2} e^{-\varphi(t)} d V_{g(t)} \\
& \quad+\frac{1}{4} \int_{M}\left|\mathscr{S}_{g(t), u(t)}+4 d u(t) \otimes d u(t)\right|_{g(t)}^{2} e^{-\varphi(t)} d V_{g(t)} \\
& \quad-\int_{M} \Delta_{g(t)}\left(\left|\nabla_{g(t)} u(t)\right|_{g(t)}^{2}\right) e^{-\varphi(t)} d V_{g(t)} .
\end{aligned}
$$


Remark 9.2. When $u \equiv 0$, this equation reduces to Li's formula [2007].

\section{The first variation of expander and shrinker entropies}

Suppose that $M$ is a closed manifold of dimension $n$. We define

$$
\mathcal{W}_{ \pm}: \bigodot_{+}^{2}(M) \times C^{\infty}(M) \times C^{\infty}(M) \times \mathbb{R}^{+} \rightarrow \mathbb{R}, \quad(g, u, f, \tau) \mapsto \mathcal{W}_{ \pm}(g, u, f, \tau)
$$

where

$$
W_{ \pm}(g, u, f, \tau):=\int_{M}\left(\tau\left(S_{g, u}+\left|\nabla_{g} f\right|_{g}^{2}\right) \mp f \pm n\right) \frac{e^{-f}}{(4 \pi \tau)^{n / 2}} d V_{g}
$$

Set

$$
\begin{aligned}
\mu_{ \pm}(g, u, \tau) & :=\inf \left\{W_{ \pm}(g, u, f, \tau) \mid f \in C^{\infty}(M), \int_{M} \frac{e^{-f}}{(4 \pi \tau)^{n / 2}} d V_{g}=1\right\}, \\
\nu_{ \pm}(g, u) & :=\sup \left\{\mu_{ \pm}(g, u, \tau) \mid \tau>0\right\} .
\end{aligned}
$$

Lemma 10.1. Suppose $v_{ \pm}(g, u)=\mathcal{W}_{ \pm}\left(g, u, f_{ \pm}, \tau_{ \pm}\right)$for some functions $f_{ \pm}$and constants $\tau_{ \pm}$satisfying

$$
\int_{M} \frac{e^{-f_{ \pm}}}{\left(4 \pi \tau_{ \pm}\right)^{n / 2}} d V_{g}=1, \quad \tau_{ \pm}>0
$$

Then we must have

$$
\begin{gathered}
\tau_{ \pm}\left(-2 \Delta_{g} f_{ \pm}+\left|\nabla_{g} f_{ \pm}\right|_{g}^{2}-S_{g, u}\right) \pm f_{ \pm} \mp n+v_{ \pm}(g, u)=0, \\
\int_{M} \frac{f_{ \pm} e^{-f_{ \pm}}}{(4 \pi \tau)^{n / 2}} d V_{g}=\frac{n}{2} \mp \nu_{ \pm}(g, u) .
\end{gathered}
$$

Proof. Since $g$ and $u$ are fixed, we consider the corresponding Lagrangian multiplier function

$$
\mathfrak{L}_{ \pm}(f, \tau ; \lambda):=\mathscr{W}_{ \pm}(g, u, f, \tau)-\lambda\left(\int_{M} \frac{e^{-f}}{(4 \pi \tau)^{n / 2}} d V_{g}-1\right) .
$$

Then the variation of $\mathfrak{L}_{ \pm}$in $f$ direction is

$$
\begin{aligned}
\delta_{f} \mathfrak{L}_{ \pm}(f, \tau ; \lambda)=\int_{M}\left(2 \tau \nabla^{i} f \nabla_{i}(\delta f) \mp \delta f+\lambda \delta f\right) \frac{e^{-f}}{(4 \pi \tau)^{n / 2}} d V_{g} & \\
& \quad-\int_{M}\left(\tau\left(S_{g, u}+\left|\nabla_{g} f\right|_{g}^{2}\right) \mp f \pm n\right) \delta f \frac{e^{-f}}{(4 \pi \tau)^{n / 2}} d V_{g} .
\end{aligned}
$$

By the divergence theorem, we calculate

$$
\begin{aligned}
\int_{M} \nabla^{i} f \cdot \nabla_{i}(\delta f) \frac{e^{-f}}{(4 \pi \tau)^{n / 2}} d V_{g} & =-\int_{M} \nabla_{i}\left(\nabla^{i} f \frac{e^{-f}}{(4 \pi \tau)^{n / 2}}\right) \delta f d V_{g} \\
& =-\int_{M}\left(\Delta_{g} f-\left|\nabla_{g} f\right|_{g}^{2}\right) \delta f \frac{e^{-f}}{(4 \pi \tau)^{n / 2}} d V_{g} .
\end{aligned}
$$


Hence

$\delta_{f} \mathfrak{L}_{ \pm}(f, \tau ; \lambda)=\int_{M}\left(\tau\left(-2 \Delta_{g} f+\left|\nabla_{g} f\right|_{g}^{2}-S_{g, u}\right) \pm f \mp n \mp 1+\lambda\right) \delta f \frac{e^{-f}}{(4 \pi \tau)^{n / 2}} d V$.

This implies that

$$
\tau_{ \pm}\left(-2 \Delta_{g} f_{ \pm}+\left|\nabla_{g} f_{ \pm}\right|_{g}^{2}-S_{g, u}\right) \pm f_{ \pm} \mp n \mp 1+\lambda_{ \pm}=0 .
$$

Since $f_{ \pm}$satisfies the normalized condition, it follows that

$$
0=\lambda_{ \pm} \mp 1+\int_{M}\left(\tau_{ \pm}\left(-2 \Delta_{g} f_{ \pm}+\left|\nabla_{g} f_{ \pm}\right|_{g}^{2}-S_{g, u}\right) \pm f_{ \pm} \mp n\right) \frac{e^{-f_{ \pm}}}{\left(4 \pi \tau_{ \pm}\right)^{n / 2}} d V_{g} .
$$

From the identity

$$
\int_{M} \Delta_{g} f \frac{e^{-f}}{(4 \pi \tau)^{n / 2}} d V_{g}=\int_{M}\left|\nabla_{g} f\right|_{g}^{2} \frac{e^{-f}}{(4 \pi \tau)^{n / 2}} d V_{g}
$$

and the definition (10-1), we obtain

$$
v_{ \pm}(g, u)=\mathcal{W}_{ \pm}\left(g, u, f_{ \pm}, \tau_{ \pm}\right)=\lambda_{ \pm} \mp 1,
$$

and, consequently,

$$
\tau_{ \pm}\left(-2 \Delta_{g} f_{ \pm}+\left|\nabla_{g} f_{ \pm}\right|_{g}^{2}-S_{g, u}\right) \pm f_{ \pm} \mp n+v_{ \pm}(g, u)=0 .
$$

The variation of $\mathfrak{L}_{ \pm}$with respect to $\tau$ indicates that

$$
\begin{aligned}
& \delta_{\tau} \mathfrak{L}_{ \pm}(f, \tau ; \lambda)= \int_{M} \delta \tau\left(S_{g, u}+\left|\nabla_{g} f\right|_{g}^{2}\right) \frac{e^{-f}}{(4 \pi \tau)^{n / 2}} d V_{g}-\lambda \int_{M}\left(-\frac{n}{2} \frac{\delta \tau}{\tau}\right) \frac{e^{-f}}{(4 \pi \tau)^{n / 2}} d V_{g} \\
& \quad+\int_{M}\left(-\frac{n}{2} \frac{\delta \tau}{\tau}\right)\left(\tau\left(S_{g, u}+\left|\nabla_{g} f\right|_{g}^{2}\right) \mp f \pm n\right) \frac{e^{-f}}{(4 \pi \tau)^{n / 2}} d V_{g} \\
&=\int_{M} \delta \tau\left(\left(1-\frac{n}{2}\right)\left(S_{g, u}+\left|\nabla_{g} f\right|_{g}^{2}\right)+\frac{n}{2 \tau}(\lambda \pm f \mp n)\right) \frac{e^{-f} d V_{g}}{(4 \pi \tau)^{n / 2}} .
\end{aligned}
$$

Using the first proved equation, we have

$$
\begin{aligned}
0 & =\int_{M}\left(\left(v_{ \pm}(g, u) \pm f_{ \pm} \mp n\right)\left(1-\frac{n}{2}\right)+\frac{n}{2}\left(v_{ \pm}(g, u) \pm f_{ \pm} \mp n \pm 1\right)\right) \frac{e^{-f_{ \pm}} d V_{g}}{\left(4 \pi \tau_{ \pm}\right)^{n / 2}} \\
& =\int_{M}\left(v_{ \pm} \pm f_{ \pm} \mp \frac{n}{2}\right) \frac{e^{-f_{ \pm}}}{\left(4 \pi \tau_{ \pm}\right)^{n / 2}} d V_{g}
\end{aligned}
$$

and therefore we obtain the second one.

For a symmetric 2-tensor $h=\left(h_{i j}\right) \in \bigodot^{2}(M)$, we set

$$
g(s):=g+s h
$$


Then the variation of $g(s)$ is

$$
\left.\frac{\partial}{\partial s}\right|_{s=0} R_{g(s)}=-h^{i j} R_{i j}+\nabla^{i} \nabla^{j} h_{i j}-\Delta_{g}\left(\operatorname{tr}_{g} h\right) .
$$

Theorem 10.2. Suppose that $(M, g)$ is a compact Riemannian manifold and $u$ a smooth function on $M$. Let $h$ be any symmetric covariant 2-tensor on $M$ and set $g(s):=g+s h$. Let $v$ be any smooth function on $M$ and $u(s):=u+s v$. If

$$
\nu_{ \pm}(g(s), u(s))=\mathcal{W}_{ \pm}\left(g(s), u(s), f_{ \pm}(s), \tau_{ \pm}(s)\right)
$$

for some smooth functions $f_{ \pm}(s)$ with

$$
\int_{M} e^{-f_{ \pm}(s)} d V /\left(4 \pi \tau_{ \pm}(s)\right)^{n / 2}=1
$$

and constants $\tau_{ \pm}(s)>0$,

$$
\begin{array}{r}
\left.\frac{d}{d s}\right|_{s=0} \nu_{ \pm}(g(s), u(s))=-\tau_{ \pm} \int_{M}\left(\left\langle h, \mathscr{Y}_{g, u}\right\rangle_{g}+\left\langle h, \nabla_{g}^{2} f\right\rangle_{g} \pm \frac{1}{2 \tau_{ \pm}} \operatorname{tr}_{g} h\right) \frac{e^{-f_{ \pm}}}{\left(4 \pi \tau_{ \pm}\right)^{n / 2}} d V_{g} \\
+4 \tau_{ \pm} \int_{M} v\left(\Delta_{g} u-\left\langle d u, d f_{ \pm}\right\rangle_{g}\right) \frac{e^{-f_{ \pm}}}{\left(4 \pi \tau_{ \pm}\right)^{n / 2}} d V_{g},
\end{array}
$$

where $f_{ \pm}:=f_{ \pm}(0)$ and $\tau_{ \pm}:=\tau_{ \pm}(0)$. In particular, the critical points of $v_{ \pm}(\cdot, \cdot)$ satisfy

$$
\mathscr{S}_{g, u}+\nabla_{g}^{2} f \pm \frac{1}{2 \tau_{ \pm}} g=0, \quad \Delta_{g} u=\left\langle d u, d f_{ \pm}\right\rangle_{g} .
$$

Consequently, if $W_{ \pm}(g, u, f, \tau)$ and $\nu_{ \pm}(g, u)$ achieve their minimums, $(M, g)$ is a gradient expanding and shrinker harmonic-Ricci soliton according to the sign.

Proof. By definition, one has

$$
\begin{aligned}
& \frac{d}{d s} v_{ \pm}(g(s), u(s)) \\
& \quad=\frac{d}{d s} W_{ \pm}\left(g(s), u(s), f_{ \pm}(s), \tau_{ \pm}(s)\right) \\
& =\int_{M}\left(\frac{\partial}{\partial s} \tau_{ \pm}(s)\left(S_{g(s), u(s)}+\left|\nabla_{g(s)} f_{ \pm}(s)\right|_{g(s)}^{2}\right)\right) \frac{e^{-f_{ \pm}(s)}}{\left(4 \pi \tau_{ \pm}(s)\right)^{n / 2}} d V_{g(s)} \\
& \quad+\int_{M}\left(\tau_{ \pm}(s) \frac{\partial}{\partial s}\left(S_{g(s), u(s)}+\left|\nabla_{g(s)} f_{ \pm}(s)\right|_{g(s)}^{2}\right) \mp \frac{\partial}{\partial s} f_{ \pm}(s)\right) \frac{e^{-f_{ \pm}(s)}}{\left(4 \pi \tau_{ \pm}(s)\right)^{n / 2}} d V_{g(s)} \\
& \quad+\int_{M}\left(\tau_{ \pm}(s)\left(S_{g(s), u(s)}+\left|\nabla_{g(s)} f_{ \pm}(s)\right|_{g(s)}^{2}\right) \mp f_{ \pm}(s) \pm n\right) \\
& \cdot \frac{\partial}{\partial s}\left(\frac{e^{-f_{ \pm}(s)}}{\left(4 \pi \tau_{ \pm}(s)\right)^{n / 2}} d V_{g(s)}\right) .
\end{aligned}
$$


Since

$$
\begin{aligned}
\frac{\partial}{\partial s} S_{g(s), u(s)} & =\frac{\partial}{\partial s} R_{g(s)}-2 \frac{\partial}{\partial s}\left|\nabla_{g(s)} u(s)\right|_{g(s)}^{2} \\
& =\frac{\partial}{\partial s} R_{g(s)}-2\left(\frac{\partial}{\partial s} g^{i j}\right) \nabla_{i} u \nabla_{j} u-4 g^{i j} \frac{\partial}{\partial s} \nabla_{i} u \cdot \nabla_{j} u \\
& =\frac{\partial}{\partial s} R_{g(s)}-2\left(-g^{i p} g^{j q} h_{p q}\right) \nabla_{i} u \nabla_{j} u-4 g^{i j} \nabla_{i}\left(\frac{\partial}{\partial s} u\right) \nabla_{j} u \\
& =\frac{\partial}{\partial s} R_{g(s)}+2 h_{p q} \nabla^{p} u \nabla^{q} u-4 \nabla_{i}\left(\frac{\partial}{\partial t} u\right) \nabla^{i} u
\end{aligned}
$$

and

$$
\begin{aligned}
& \frac{\partial}{\partial s}\left(\frac{e^{-f_{ \pm}(s)}}{\left(4 \pi \tau_{ \pm}(s)\right)^{n / 2}} d V_{g(s)}\right) \\
& =\left(-\frac{\partial}{\partial s} f_{ \pm}(s)-\frac{n}{2 \tau_{ \pm}(s)} \frac{\partial}{\partial s} \tau_{ \pm}(s)\right) \frac{e^{-f_{ \pm}(s)}}{\left(4 \pi \tau_{ \pm}(s)\right)^{n / 2}} d V_{g(s)}+\frac{e^{-f_{ \pm}(s)}}{\left(4 \pi \tau_{ \pm}(s)\right)^{n / 2}} \frac{\partial}{\partial s} d V_{g(s)} \\
& =\left(-\frac{\partial}{\partial s} f_{ \pm}(s)-\frac{n}{2 \tau_{ \pm}(s)} \frac{\partial}{\partial s} \tau_{ \pm}(s)+\frac{1}{2} \operatorname{tr}_{g} h\right) \frac{e^{-f_{ \pm}(s)}}{\left(4 \pi \tau_{ \pm}(s)\right)^{n / 2}} d V_{g(s)},
\end{aligned}
$$

it follows that

$$
\begin{aligned}
& \frac{d}{d s} v_{ \pm}(g(s), u(s)) \\
& =\int_{M} \frac{\partial}{\partial s} \tau_{ \pm}(s)\left(S_{g(s), u(s)}+\left|\nabla_{g(s)} f_{ \pm}(s)\right|_{g(s)}^{2}\right) \frac{e^{-f_{ \pm}(s)}}{\left(4 \pi \tau_{ \pm}(s)\right)^{n / 2}} d V_{g(s)} \\
& \quad+\int_{M}\left(\tau _ { \pm } ( s ) \left(\frac{\partial}{\partial s} R_{g(s)}+2 h_{p q} \nabla^{p} u \nabla^{q} u-4 \nabla_{i}\left(\frac{\partial}{\partial s} u\right) \nabla^{i} u\right.\right. \\
& \left.\left.\quad-h_{p q} \nabla^{p} f \nabla^{q} f+2 \nabla_{i}\left(\frac{\partial}{\partial s} f\right) \nabla^{i} f\right) \mp \frac{\partial}{\partial s} f_{ \pm}(s)\right) \frac{e^{-f_{ \pm}(s)}}{\left(4 \pi \tau_{ \pm}(s)\right)^{n / 2}} d V_{g(s)} \\
& \quad+\int_{M}\left(-\frac{\partial}{\partial s} f_{ \pm}(s)-\frac{n}{2 \tau_{ \pm}(s)} \frac{\partial}{\partial s} \tau_{ \pm}(s)+\frac{1}{2} \operatorname{tr}_{g} h\right) \\
& \quad \cdot\left(\tau_{ \pm}(s)\left(S_{g(s), u(s)}+\left|\nabla_{g(s)} f_{ \pm}(s)\right|_{g(s)}^{2}\right) \mp f_{ \pm}(s) \pm n\right) \frac{e^{-f_{ \pm}(s)}}{\left(4 \pi \tau_{ \pm}(s)\right)^{n / 2}} d V_{g(s)} .
\end{aligned}
$$

From the equalities

$$
\begin{aligned}
\int_{M} \Delta_{g} \operatorname{tr}_{g} h \cdot e^{-f} d V_{g} & =\int_{M} \operatorname{tr}_{g} h \cdot \Delta_{g}\left(e^{-f}\right) d V_{g} \\
& =\int_{M} \operatorname{tr}_{g} h\left(-\Delta_{g} f+\left|\nabla_{g}, f\right|_{g}^{2}\right) e^{-f} d V_{g},
\end{aligned}
$$




$$
\begin{aligned}
\int_{M} \nabla^{i} \nabla^{j} h_{i j} \cdot e^{-f} d V_{g} & =\int_{M} h_{i j} \nabla^{i} \nabla^{j}\left(e^{-f}\right) d V \\
& =\int_{M} h_{i j}\left(-\nabla^{i} \nabla^{j} f+\nabla^{i} f \nabla^{j} f\right) e^{-f} d V_{g}, \\
\int_{M} \nabla_{i}\left(\frac{\partial}{\partial s} f\right) \nabla^{i} f e^{-f} d V_{g} & =\int_{M}-\frac{\partial}{\partial s} f\left(\Delta_{g} f-\left|\nabla_{g} f\right|_{g}^{2}\right) e^{-f} d V_{g}, \\
\int_{M} \Delta_{g}\left(e^{-f}\right) d V_{g} & =\int_{M}\left(-\Delta_{g} f+\left|\nabla_{g} f\right|_{g}^{2}\right) e^{-f} d V_{g},
\end{aligned}
$$

and Lemma 10.1, we obtain

$$
\begin{aligned}
\left.\frac{d}{d s}\right|_{s=0} & \nu_{ \pm}(g(s), u(s)) \\
= & \left.\int_{M} \frac{\partial}{\partial s}\right|_{s=0} \tau_{ \pm}(s)\left(S_{g, u}+\left|\nabla_{g} f\right|_{g}^{2}\right) \frac{e^{-f_{ \pm}}}{\left(4 \pi \tau_{ \pm}\right)^{n / 2}} d V_{g} \\
& +\int_{M}\left(\tau _ { \pm } \left(-h^{i j} R_{i j}+\nabla^{i} \nabla_{j} h_{i j}-\Delta_{g}\left(\operatorname{tr}_{g} h\right)+2 h_{p q} \nabla^{p} u \nabla^{q} u\right.\right. \\
& \left.\left.\quad-4 \nabla_{i} v \nabla^{i} u-h_{p q} \nabla^{p} f \nabla^{q} f+2 \nabla_{i}\left(\left.\frac{\partial}{\partial s}\right|_{s=0} f(s)\right) \nabla^{i} f\right)\left.\mp \frac{\partial}{\partial s}\right|_{s=0} f(s)\right) \\
& \quad \frac{e^{-f_{ \pm}}}{\left(4 \pi \tau_{ \pm}\right)^{n / 2}} d V_{g}+\int_{M}\left(-\left.\frac{\partial}{\partial s}\right|_{s=0} f_{ \pm}(s)-\left.\frac{n}{2 \tau_{ \pm}}(s) \frac{\partial}{\partial s}\right|_{s=0} \tau_{ \pm}(s)+\frac{1}{2} \operatorname{tr}_{g} h\right) \\
& \cdot\left(\tau_{ \pm}\left(S_{g, u}+\left|\nabla_{g} f_{ \pm}\right|_{g}^{2}\right) \mp f_{ \pm} \pm n\right) \frac{e^{-f_{ \pm}}}{\left(4 \pi \tau_{ \pm}\right)^{n / 2}} d V_{g} .
\end{aligned}
$$

If we denote by $B$ the last term and by $A$ the remaining terms,

$$
\begin{aligned}
A=\int_{M}\left(\left.\frac{\partial}{\partial s}\right|_{s=0}\right. & \tau_{ \pm}(s)\left(\left|\nabla_{g} f_{ \pm}\right|_{g}^{2}+S_{g, u}\right) \\
& \left.-\tau_{ \pm}\left(h^{i j} \nabla_{i} \nabla_{j} f_{ \pm}+h^{i j} S_{i j}+4 \nabla_{i} v \cdot \nabla^{i} u\right) \mp \frac{\partial}{\partial s} f_{ \pm}\right) \frac{e^{-f_{ \pm}}}{\left(4 \pi \tau_{ \pm}\right)^{n / 2}} d V_{g} \\
& +\int_{M} \tau_{ \pm}\left(\Delta_{g} f_{ \pm}-\left|\nabla_{g} f_{ \pm}\right|_{g}^{2}\right)\left(\operatorname{tr}_{g} h-\left.2 \frac{\partial}{\partial s}\right|_{s=0} f(s)\right) \frac{e^{-f_{ \pm}}}{\left(4 \pi \tau_{ \pm}\right)^{n / 2}} d V_{g} .
\end{aligned}
$$

The normalized condition

$$
1=\int_{M} \frac{e^{-f_{ \pm}(s)}}{\left(4 \pi \tau_{ \pm}(s)\right)^{n / 2}} d V_{g}
$$

implies

$$
0=\int_{M}\left(-\left.\frac{\partial}{\partial s}\right|_{s=0} f_{ \pm}(s)-\left.\frac{n}{2 \tau_{ \pm}} \frac{\partial}{\partial s}\right|_{s=0} \tau_{ \pm}(s)+\frac{1}{2} \operatorname{tr}_{g} h\right) \frac{e^{-f_{ \pm}(s)}}{\left(4 \pi \tau_{ \pm}(s)\right)^{n / 2}} d V_{g} .
$$


From Lemma 10.1, we conclude that

$$
\tau_{ \pm} S_{g, u}-\tau_{ \pm}\left(\left|\nabla_{g} f_{ \pm}\right|_{g}^{2}-2 \Delta_{g} f_{ \pm}\right)= \pm f_{ \pm} \mp n+v_{ \pm}(g, u) .
$$

Therefore

$$
\tau_{ \pm}\left(S_{g, u}+\left|\nabla_{g} f_{ \pm}\right|_{g}^{2}\right) \mp f_{ \pm} \pm n=2 \tau_{ \pm}\left(\left|\nabla_{g} f_{ \pm}\right|_{g}^{2}-\Delta_{g} f_{ \pm}\right)+v_{ \pm}(g, u) .
$$

Plugging this into the definition of $B$ yields

$$
\begin{aligned}
& B= \int_{M}\left(-\left.\frac{\partial}{\partial s}\right|_{s=0} f_{ \pm}(s)-\left.\frac{n}{2 \tau_{ \pm}} \frac{\partial}{\partial s}\right|_{s=0} \tau_{ \pm}(s)+\frac{1}{2} \operatorname{tr}_{g} h\right) \\
& \cdot\left(2 \tau_{ \pm}\left(\left|\nabla_{g} f_{ \pm}\right|_{g}^{2}-\Delta_{g} f_{ \pm}\right)+v_{ \pm}(g, u)\right) \frac{e^{-f_{ \pm}}}{\left(4 \pi \tau_{ \pm}\right)^{n / 2}} d V_{g} \\
&= \int_{M}\left(-\left.\frac{\partial}{\partial s}\right|_{s=0} f_{ \pm}(s)-\left.\frac{n}{2 \tau_{ \pm}} \frac{\partial}{\partial s}\right|_{s=0} \tau_{ \pm}(s)+\frac{1}{2} \operatorname{tr}_{g} h\right) \\
&=\int_{M}\left(-\left.\frac{\partial}{\partial s}\right|_{s=0} f_{ \pm}(s)+\frac{1}{2} \operatorname{tr}_{g} h\right) 2 \tau_{ \pm}\left(\left|\nabla_{g} f_{ \pm}\right|_{g}^{2}-\Delta_{g} f_{ \pm}\right) \frac{e^{-f_{ \pm}}}{\left(4 \pi \tau_{ \pm}\right)^{n / 2}} d V_{g},
\end{aligned}
$$

where we use the fact that

$$
\int_{M} \Delta_{g}\left(e^{-f}\right) d V_{g}=0
$$

Hence $B$ cancels with the last term in $A$. Therefore the above variation equals

$$
\begin{aligned}
\left.\frac{d}{d s}\right|_{s=0} \nu_{ \pm}(g(s), u(s)) & \\
=\int_{M}\left(\left.\frac{\partial}{\partial s}\right|_{s=0} \tau_{ \pm}(s)(\right. & \left.\left|\nabla_{g} f_{ \pm}\right|_{g}^{2}+S_{g, u} \pm \frac{n}{2 \tau_{ \pm}}\right)-\tau_{ \pm}\left(h^{i j} \nabla_{i} \nabla_{j} f+h^{i j} S_{i j}\right. \\
& \left.\left. \pm \frac{1}{2 \tau_{ \pm}} \operatorname{tr}_{g} h+4 v\left(\langle d u, d f\rangle-\Delta_{g} u\right)\right)\right) \frac{e^{-f_{ \pm}}}{\left(4 \pi \tau_{ \pm}\right)^{n / 2}} d V_{g} .
\end{aligned}
$$

To prove the theorem, it is sufficient to show that

$$
\int_{M}\left(\left|\nabla_{g} f_{ \pm}\right|_{g}^{2}+S_{g, u} \pm \frac{n}{2 \tau_{ \pm}}\right) \frac{e^{-f_{ \pm}}}{\left(4 \pi \tau_{ \pm}\right)^{n / 2}} d V=0
$$

Since $M$ is compact, we have

$$
0=\int_{M} \Delta_{g}\left(e^{-f_{ \pm}}\right)=\int_{M}\left(-\Delta_{g} f_{ \pm}+\left|\nabla_{g} f_{ \pm}\right|_{g}^{2}\right) e^{-f_{ \pm}} d V
$$


Hence

$$
\begin{aligned}
\int_{M}\left(\left|\nabla_{g} f_{ \pm}\right|^{2}+S_{g, u} \pm \frac{n}{2 \tau_{ \pm}}\right) & \frac{e^{-f_{ \pm}}}{\left(4 \pi \tau_{ \pm}\right)^{n / 2}} d V \\
& =\int_{M}\left(2 \Delta_{g} f_{ \pm}-\left|\nabla_{g} f\right|_{g}^{2}+S_{g, u} \pm \frac{n}{2 \sigma_{ \pm}}\right) \frac{e^{-f_{ \pm}}}{\left(4 \pi \tau_{ \pm}\right)^{n / 2}} d V .
\end{aligned}
$$

Lemma 10.1 now indicates

$$
\begin{aligned}
& \int_{M}\left(\left|\nabla_{g} f_{ \pm}\right|^{2}+S_{g, u} \pm \frac{n}{2 \tau_{ \pm}}\right) \frac{e^{-f_{ \pm}}}{\left(4 \pi \tau_{ \pm}\right)^{n / 2}} d V \\
& =\int_{M}\left(\frac{ \pm f_{ \pm} \mp n+v_{ \pm}(g, u)}{\tau_{ \pm}} \pm \frac{n}{2}\right) \frac{e^{-f_{ \pm}}}{\left(4 \pi \tau_{ \pm}\right)^{n / 2}} d V \\
& =\int_{M} \frac{1}{\tau_{ \pm}}\left( \pm f_{ \pm} \mp \frac{n}{2}+v_{ \pm}(g, u)\right) \frac{e^{-f_{ \pm}}}{\left(4 \pi \tau_{ \pm}\right)^{n / 2}} d V \\
& =\frac{1}{\tau_{ \pm}}\left( \pm \frac{n}{2}-v_{ \pm}(g, u) \mp \frac{n}{2}+v_{ \pm}(g, u)\right)=0 \text {. }
\end{aligned}
$$

The sign + corresponds to the gradient expanding soliton and the sign - to the gradient shrinker soliton.

Corollary 10.3. Suppose that $(M, g)$ is a compact Riemannian manifold and $u$ is a smooth function on $M$. Let $h$ be any symmetric covariant 2-tensor on $M$ and set $g(s):=g+s h$. Let $v$ be any smooth function on $M$ and $u(s):=u+s v$. If $v_{ \pm}(g(s), u(s))=W_{ \pm}\left(g(s), u(s), f_{ \pm}(s), \tau_{ \pm}(s)\right)$ for some smooth function $f_{ \pm}(s)$ with $\int_{M} e^{-f_{ \pm}(s)} d V /\left(4 \pi \tau_{ \pm}(s)\right)^{n / 2}=1$ and a constant $\tau_{ \pm}(s)>0$, and $(g, u)$ is a critical point of $v_{ \pm}(\cdot, \cdot)$, then

$$
\mathscr{Y}_{g, u}=\mp \frac{1}{2 \tau_{ \pm}} g, \quad f_{ \pm} \equiv \text { constant }
$$

Thus, if $\mathcal{W}_{ \pm}(g, u, \cdot, \cdot)$ achieve their minimum and $(g, u)$ is a critical point of $v_{ \pm}(\cdot, \cdot),(M, g, u)$ satisfies the static Einstein vacuum equation.

Proof. According to Lemma 10.1 and Theorem 10.2, we have

$$
\begin{aligned}
& \tau_{ \pm}\left(-2 \Delta_{g} f_{ \pm}+\left|\nabla_{g} f_{ \pm}\right|_{g}^{2}-S_{g, u}\right) \pm f_{ \pm} \mp n \\
& \quad=-v_{ \pm}=-\int_{M}\left(\tau_{ \pm}\left(S_{g, u}+\left|\nabla_{g} f\right|_{g}^{2}\right) \mp f_{ \pm} \pm n\right) \frac{e^{-f_{ \pm}}}{\left(4 \pi \tau_{ \pm}\right)^{n / 2}} d V_{g}
\end{aligned}
$$


and hence

$$
\begin{aligned}
2 \Delta_{g} f_{ \pm}-\left|\nabla_{g} f_{ \pm}\right|_{g}^{2}+S_{g, u} & =\int_{M}\left(S_{g, u}+\left|\nabla_{g} f_{ \pm}\right|_{g}^{2}\right) \frac{e^{-f_{ \pm}}}{\left(4 \pi \tau_{ \pm}\right)^{n / 2}} d V_{g} \\
& =\int_{M}\left(S_{g, u}+\Delta_{g} f_{ \pm}\right) \frac{e^{-f_{ \pm}}}{\left(4 \pi \tau_{ \pm}\right)^{n / 2}} d V_{g} \\
& =\mp \frac{n}{2 \tau_{ \pm}}=S_{g, u}+\Delta_{g} f_{ \pm} .
\end{aligned}
$$

From this we get $\Delta_{g} f_{ \pm}=\left|\nabla_{g} f_{ \pm}\right|_{g}^{2}$. After integrating on both sides, the functions $f_{ \pm}$must be constant, which implies $\mathscr{S}_{g} \pm\left(1 /\left(2 \tau_{ \pm}\right)\right) g=0$.

Remark 10.4. In the situation of Corollary 10.3 , by normalization, we my choose $f_{ \pm}=n / 2$.

\section{Acknowledgements}

The author thanks Professor Kefeng Liu, who teaches the author mathematics, Professor Hongwei $\mathrm{Xu}$, and other staff members at the Center of Mathematical Science, where part of this work was done.

\section{References}

[Cao 2007] X. Cao, "Eigenvalues of $(-\Delta+R / 2)$ on manifolds with nonnegative curvature operator", Math. Ann. 337:2 (2007), 435-441. MR 2007g:53071 Zbl 1105.53051

[Cao 2008] X. Cao, "First eigenvalues of geometric operators under the Ricci flow", Proc. Amer. Math. Soc. 136:11 (2008), 4075-4078. MR 2009f:53098 Zbl 1166.58007

[He et al. 2008] C.-L. He, S. Hu, D.-X. Kong, and K. Liu, "Generalized Ricci flow. I. Local existence and uniqueness", pp. 151-171 in Topology and physics, Nankai Tracts Math. 12, World Sci. Publ., Hackensack, NJ, 2008. MR 2010k:53098

[Li 2007] J.-F. Li, "Eigenvalues and energy functionals with monotonicity formulae under Ricci flow”, Math. Ann. 338:4 (2007), 927-946. MR 2008c:53068 Zbl 1127.53059

[Li 2012] Y. Li, "Generatlized Ricci flow I: higher dericative estimates for compact manifolds", Analysis \& PDE 5:4 (2012), 747-775.

[List 2006] B. List, Evolution of an extended Ricci flow system, Ph.D. thesis, Fachbereich Mathematik und Informatik der Freie Universität Berlin, 2006, Available at http://www.diss.fu-berlin.de/2006/ 180/index.html.

[Müller 2012] R. Müller, "Ricci flow coupled with harmonic map flow", Ann. Sci. Éc. Norm. Supér. (4) 45:1 (2012), 101-142. MR 2961788

[Oliynyk et al. 2006] T. Oliynyk, V. Suneeta, and E. Woolgar, "A gradient flow for worldsheet nonlinear sigma models”, Nuclear Phys. B 739:3 (2006), 441-458. MR 2006m:81185 Zbl 1109.81058

[Streets 2007] J. D. Streets, Ricci Yang-Mills flow, Ph.D. thesis, Duke University, 2007, Available at http://www.math.uci.edu/ jstreets/papers/StreetsThesis.pdf.

[Streets 2008] J. Streets, "Regularity and expanding entropy for connection Ricci flow", J. Geom. Phys. 58:7 (2008), 900-912. MR 2009f:53105 Zbl 1144.53326 
[Streets 2009] J. Streets, "Singularities of renormalization group flows", J. Geom. Phys. 59:1 (2009), 8-16. MR 2010a:53143 Zbl 1153.53329

[Streets 2010] J. Streets, "Ricci Yang-Mills flow on surfaces", Adv. Math. 223:2 (2010), 454-475. MR 2011c:53164 Zbl 1190.53069

[Young 2008] A. N. Young, Modified Ricci flow on a principal bundle, Ph.D. thesis, The University of Texas at Austin, 2008, Available at http://search.proquest.com/docview/193674070.

Received August 27, 2012. Revised January 6, 2013.

YI LI

DEPARTMENT OF MATHEMATICS

JOHNS HOPKINS UNIVERSITY

3400 N. CHARLES STREET

BALTIMORE, MD 21218

UNITED STATES

yli@math.jhu.edu

Current address:

DEPARTMENT OF MATHEMATICS

SHANGHAi JiaO TONG UNIVERSITY

800 Dong Chuan RoAd, Min Hang District

SHANGHAI, 200240

CHINA 


\title{
PACIFIC JOURNAL OF MATHEMATICS
}

\author{
msp.org/pjm
}

Founded in 1951 by E. F. Beckenbach (1906-1982) and F. Wolf (1904-1989)

\section{EDITORS}

V. S. Varadarajan (Managing Editor)

Department of Mathematics

University of California

Los Angeles, CA 90095-1555

pacific@math.ucla.edu

Paul Balmer

Department of Mathematics

University of California

Los Angeles, CA 90095-1555

balmer@math.ucla.edu

Daryl Cooper

Department of Mathematics

University of California

Santa Barbara, CA 93106-3080 cooper@math.ucsb.edu

Jiang-Hua $\mathrm{Lu}$

Department of Mathematics

Pokfulam Rd., Hong Kong jhlu@maths.hku.hk
The University of Hong Kong

Don Blasius

Department of Mathematics University of California

Los Angeles, CA 90095-1555

blasius@math.ucla.edu

Robert Finn

Department of Mathematics Stanford University

Stanford, CA 94305-2125

finn@math.stanford.edu

Sorin Popa

Department of Mathematics

University of California

Los Angeles, CA 90095-1555 popa@math.ucla.edu

Paul Yang

Department of Mathematics Princeton University

Princeton NJ 08544-1000

yang@math.princeton.edu

\section{PRODUCTION}

Silvio Levy, Scientific Editor, production@msp.org

\section{SUPPORTING INSTITUTIONS}

ACADEMIA SINICA, TAIPEI

CALIFORNIA INST. OF TECHNOLOGY

INST. DE MATEMÁTICA PURA E APLICADA

KEIO UNIVERSITY

MATH. SCIENCES RESEARCH INSTITUTE

NEW MEXICO STATE UNIV.

OREGON STATE UNIV.

\author{
STANFORD UNIVERSITY \\ UNIV. OF BRITISH COLUMBIA \\ UNIV. OF CALIFORNIA, BERKELEY \\ UNIV. OF CALIFORNIA, DAVIS \\ UNIV. OF CALIFORNIA, LOS ANGELES \\ UNIV. OF CALIFORNIA, RIVERSIDE \\ UNIV. OF CALIFORNIA, SAN DIEGO \\ UNIV. OF CALIF., SANTA BARBARA
}

\author{
Vyjayanthi Chari \\ Department of Mathematics \\ University of California \\ Riverside, CA 92521-0135 \\ chari@math.ucr.edu \\ Kefeng Liu \\ Department of Mathematics \\ University of California \\ Los Angeles, CA 90095-1555 \\ liu@math.ucla.edu \\ Jie Qing \\ Department of Mathematics \\ University of California \\ Santa Cruz, CA 95064 \\ qing@cats.ucsc.edu
}

These supporting institutions contribute to the cost of publication of this Journal, but they are not owners or publishers and have no responsibility for its contents or policies.

See inside back cover or msp.org/pjm for submission instructions.

The subscription price for 2014 is US \$410/year for the electronic version, and \$535/year for print and electronic.

Subscriptions, requests for back issues and changes of subscribers address should be sent to Pacific Journal of Mathematics, P.O. Box 4163, Berkeley, CA 94704-0163, U.S.A. The Pacific Journal of Mathematics is indexed by Mathematical Reviews, Zentralblatt MATH, PASCAL CNRS Index, Referativnyi Zhurnal, Current Mathematical Publications and Web of Knowledge (Science Citation Index).

The Pacific Journal of Mathematics (ISSN 0030-8730) at the University of California, c/o Department of Mathematics, 798 Evans Hall \#3840, Berkeley, CA 94720-3840, is published twelve times a year. Periodical rate postage paid at Berkeley, CA 94704, and additional mailing offices. POSTMASTER: send address changes to Pacific Journal of Mathematics, P.O. Box 4163, Berkeley, CA 94704-0163.

PJM peer review and production are managed by EditFLOW ${ }^{\circledR}$ from Mathematical Sciences Publishers.

\section{PUBLISHED BY}

mathematical sciences publishers

nonprofit scientific publishing

http://msp.org/

(C) 2014 Mathematical Sciences Publishers 


\section{PACIFIC JOURNAL OF MATHEMATICS}

Volume $267 \quad$ No. $1 \quad$ January 2014

Numerical study of unbounded capillary surfaces

YASUNORI AOKI and HANS DE STERCK

Dual $R$-groups of the inner forms of $\operatorname{SL}(N)$

KUOK FAI CHAO and WEN-WEI LI

Automorphisms and quotients of quaternionic fake quadrics

AMIR DŽAmBIĆ and XAVIER ROULLEAU

Distance of bridge surfaces for links with essential meridional spheres 121

YEONHEE JANG

Normal states of type III factors

YASUYUKI KAWAHIGASHI, YOSHIKO OGATA and ERLING

STØRMER

Eigenvalues and entropies under the harmonic-Ricci flow

YI LI

Quantum extremal loop weight modules and monomial crystals

MATHIEU MANSUY

Lefschetz fibrations with small slope

NAOYUKI MONDEN 